\title{
KLF4 protein stability regulated by interaction with pluripotency transcription factors overrides transcriptional control
}

\author{
Navroop K. Dhaliwal, Luis E. Abatti, and Jennifer A. Mitchell \\ Department of Cell and Systems Biology, University of Toronto, Toronto, Ontaria M5S3G5, Canada
}

Embryonic stem (ES) cells are regulated by a network of transcription factors that maintain the pluripotent state. Differentiation relies on down-regulation of pluripotency transcription factors disrupting this network. While investigating transcriptional regulation of the pluripotency transcription factor Kruppel-like factor 4 (Klf4), we observed that homozygous deletion of distal enhancers caused a 17-fold decrease in Klf4 transcript but surprisingly decreased protein levels by less than twofold, indicating that posttranscriptional control of KLF4 protein overrides transcriptional control. The lack of sensitivity of KLF4 to transcription is due to high protein stability (half-life $>24$ h). This stability is context-dependent and is disrupted during differentiation, as evidenced by a shift to a half-life of $<2 \mathrm{~h}$. KLF4 protein stability is maintained through interaction with other pluripotency transcription factors (NANOG, SOX2, and STAT3) that together facilitate association of KLF4 with RNA polymerase II. In addition, the KLF4 DNA-binding and transactivation domains are required for optimal KLF4 protein stability. Posttranslational modification of KLF4 destabilizes the protein as cells exit the pluripotent state, and mutations that prevent this destabilization also prevent differentiation. These data indicate that the core pluripotency transcription factors are integrated by posttranslational mechanisms to maintain the pluripotent state and identify mutations that increase KLF4 protein stability while maintaining transcription factor function.

[Keywords: KLF4; pluripotency; protein stability; stem cells; transcription; ubiquitin]

Supplemental material is available for this article.

Received January 16, 2019; revised version accepted May 13, 2019.

Differentiation of embryonic stem (ES) cells requires disruption to the regulatory network that maintains pluripotency gene expression. Kruppel-like factor 4 (KLF4), a member of the Kruppel-like factor family of conserved zinc finger transcription factors, is known to interact with the core network of pluripotency transcription factors (NANOG, SOX2, and OCT4) in order to regulate genes required for maintenance of pluripotency and reprogramming (Wei et al. 2009, 2013; Zhang et al. 2010; Xie et al. 2017; Dhaliwal et al. 2018). Sustained expression of constitutively nuclear KLF4 prevents differentiation of ES cells, indicating that a loss of KLF4 protein function is required for differentiation (Dhaliwal et al. 2018). It is generally accepted that differentiation occurs due to a loss of pluripotency transcription factor activity at enhancers that disrupts pluripotency gene expression and allows for the expression of differentiation-associated genes; however, the molecular mechanisms through which this disruption occurs are not well defined.

Genome-wide binding of transcription factors and coactivators can identify enhancers required for gene tran-

Corresponding author: ja.mitchell@utoronto.ca

Article published online ahead of print. Article and publication date are online at http://www.genesdev.org/cgi/doi/10.1101/gad.324319.119. scription in a particular cellular context (Chen et al. 2008, 2012; Visel et al. 2009; Moorthy et al. 2017). These enhancers are often located multikilobase distances from the genes that they regulate and form physical loops with their target gene promoters (Carter et al. 2002; Tolhuis et al. 2002; Schoenfelder et al. 2015). In pluripotent ES cells, specific enhancers have been identified that regulate Sox2, Klf4, Nanog, and Oct4 (Pou5f1) at the transcriptional level (Yeom et al. 1996; Li et al. 2014; Zhou et al. 2014; Blinka et al. 2016; Xie et al. 2017). In addition to these intrinsic mechanisms that regulate the expression of pluripotency transcription factors, the balance between maintaining the pluripotent state and inducing differentiation is also modulated by cell-extrinsic factors and cell signaling cascades. Leukemia inhibitory factor (LIF) maintains the pluripotent state by activating JAKSTAT signaling, causing phosphorylation and activation of STAT3 (Niwa et al. 1998; Matsuda et al. 1999). Activated STAT3 induces transcription of Klf4 through binding to

(C) 2019 Dhaliwal et al. This article is distributed exclusively by Cold Spring Harbor Laboratory Press for the first six months after the full-issue publication date (see http://genesdev.cshlp.org/site/misc/terms.xhtml). After six months, it is available under a Creative Commons License (Attribution-NonCommercial 4.0 International), as described at http://creativecommons.org/licenses/by-nc/4.0/. 
the enhancers downstream from Klf4 (Hall et al. 2009; Zhang et al. 2010; Xie et al. 2017). In addition, dual inhibition ( $2 \mathrm{i}$; GSK3 and MEK inhibition) maintains ES cells in a naïve state closest to that of the precursor cells from the pluripotent epiblast of preimplantation embryos (Nichols and Smith 2009; Wray et al. 2010; Tosolini and Jouneau 2016).

As pluripotency master regulators are transcription factors, reduced transcription of specific genes is generally considered the mechanism through which differentiation of ES cells occurs; however, changes in gene transcription do not always correlate with changes in protein levels. At a genome scale, evaluation of the correlation between mRNA abundance and protein abundance estimates that, for cells in a steady state, $50 \%-80 \%$ of the variability in protein levels can be explained by the levels of mRNA present (for review, see Liu et al. 2016). For cells undergoing dynamic transitions (for example, during monocyteto-macrophage differentiation), protein and mRNA levels become decoupled during the early differentiation phase, mainly due to a delay in translation compared with transcription (Kristensen et al. 2013). In both cases, exceptions exist where mRNA and protein levels do not correlate even when delays in translation are taken into account; however, the mechanism through which this occurs is not well understood. Transcription factors generally display low protein stability, which allows rapid cell state transitions (Hochstrasser and Varshavsky 1990; Zhou et al. 2004; Jovanovic et al. 2015). In this study, however, we show that KLF4 protein levels are highly decoupled from the RNA levels due to the exceptional stability of the KLF4 protein in naïve ES cells maintained in LIF/2i. Homozygous deletion of downstream $\mathrm{Klf4}$ enhancer regions caused a 17-fold reduction in Klf4 transcript levels, whereas KLF4 protein levels were reduced by less than twofold. Surprisingly, we observed a greater reduction of KLF4 protein levels (greater than threefold) in ES cells with compromised SOX2 expression despite the observation that Klf4 transcript levels are unchanged in these cells. We found that these discrepancies in KLF4 protein and transcript levels are due to modulation of KLF4 protein stability by SOX2, NANOG, and activated STAT3 as well as domains within the KLF4 protein that anchor KLF4 in the nucleus. During pluripotency exit, KLF4 protein becomes destabilized. Preventing this destabilization through mutation of KLF4-destabilizing motifs blocks pluripotency exit. The core pluripotency maintenance transcription factors are known to function in a highly integrated way to maintain transcriptional control of the pluripotent state. Here we show a new way in which these factors regulate each other that bypasses transcriptional control but maintains posttranslational control of KLF4 function.

\section{Results}

Klf4 transcript and protein levels are uncoupled in ES cells maintained in LIF/2i

For ES cells maintained in LIF/serum, Klf4 has been shown to be regulated by three enhancers 54-68 kb down- stream from the gene; deletion of this region was found to reduce $\mathrm{Klf} 4$ transcription by $90 \%$, greatly affecting KLF4 protein levels (Xie et al. 2017). For ES cells maintained in the more naïve state by LIF/ $2 \mathrm{i}$, we determined that although the enhancers remain important for maintaining transcript levels, functional KLF4 protein is maintained in the absence of the enhancers. We used F1 (Mus musculus ${ }^{129} \times$ Mus castaneus) ES cells, allowing allele-specific deletion screening and gene expression analysis (Zhou et al. 2014; Moorthy and Mitchell 2016). Upon deletion of two $(\Delta 1$, eightfold reduction in RNA) or all three $(\Delta 2$, 17-fold reduction in RNA) enhancers, we observed a dramatic reduction in Klf4 transcript levels but a much more subtle change in KLF4 protein levels (Fig. 1; Supplemental Fig. S1). KLF4 protein levels are significantly reduced only in cells with the $\Delta 2$ homozygous deletion $\left(\Delta 2^{129 / \text { Cast }}\right)$, and, in these cells, which displayed a 17 -fold reduction in mRNA, protein was reduced by less than twofold. To confirm that this was not an effect of recent enhancer deletion, we investigated Klf4 transcript and protein levels in cells maintained to later passages (P9) but identified no significant differences between early and late passages. Transcript and protein levels of other pluripotency transcription factors (Oct4, Sox2, Nanog, Klf2, and Klf5) remained unchanged in Klf4 enhancer-deleted clones (Fig. 1; Supplemental Fig. S1).

Surprisingly, we observed that KLF4 protein levels were significantly reduced by greater than threefold in clones with reduced SOX2 protein levels due to a homozygous deletion of the enhancer that regulates Sox2 transcription in ES cells $\left(\Delta S_{C R}{ }^{129 / C a s t}\right)$ (Li et al. 2014; Zhou et al. 2014) despite the observation that Klf4 transcript levels are unaffected by SCR deletion (Fig. 1A). To examine the levels of functional nuclear KLF4 protein, we used proximity ligation amplification (PLA) to investigate the interaction between KLF4 and active Ser5 phosphorylated RNA polymearase II (RNAPII-S5P) in individual nuclei. Similar to the results for total KLF4 protein levels, we found only a subtle reduction in KLF4/RNAPII interaction in cells with the Klf4 enhancer deletions; however, we observed that the greatest reduction in KLF4 association with RNAPII occurred in cells with reduced SOX2 protein levels $\left(\Delta S_{C R}{ }^{129 / \text { Cast }}\right)$ (Fig. 1C). Together, these data reveal a disconnect between the levels of Klf4 RNA and protein in naïve ES cells and indicate that the KLF4 protein may be highly stable in the naïve pluripotent state.

\section{KLF4 protein stability is regulated by LIF and MAPK signaling pathways}

Investigation of KLF4 protein stability in naïve ES cells maintained in LIF/2 $\mathrm{i}$ and cells differentiated for $24 \mathrm{~h}$ revealed that KLF4 protein is more stable in undifferentiated cells with a $t_{1 / 2}>24 \mathrm{~h}$. After removal of LIF/2i for $24 \mathrm{~h}$, KLF4 becomes unstable with a $t_{1 / 2}<2 \mathrm{~h}$ (Fig. 2A). In contrast, the other pluripotency transcription factors (OCT4, SOX2, and NANOG) are unstable $\left(t_{1 / 2} 2-4 \mathrm{~h}\right)$ in undifferentiated cells, and their stability was not affected by differentiation (Supplemental Fig. S2). Similarly, the other ES cellexpressed Klf family proteins (KLF2 and KLF5) are not as 


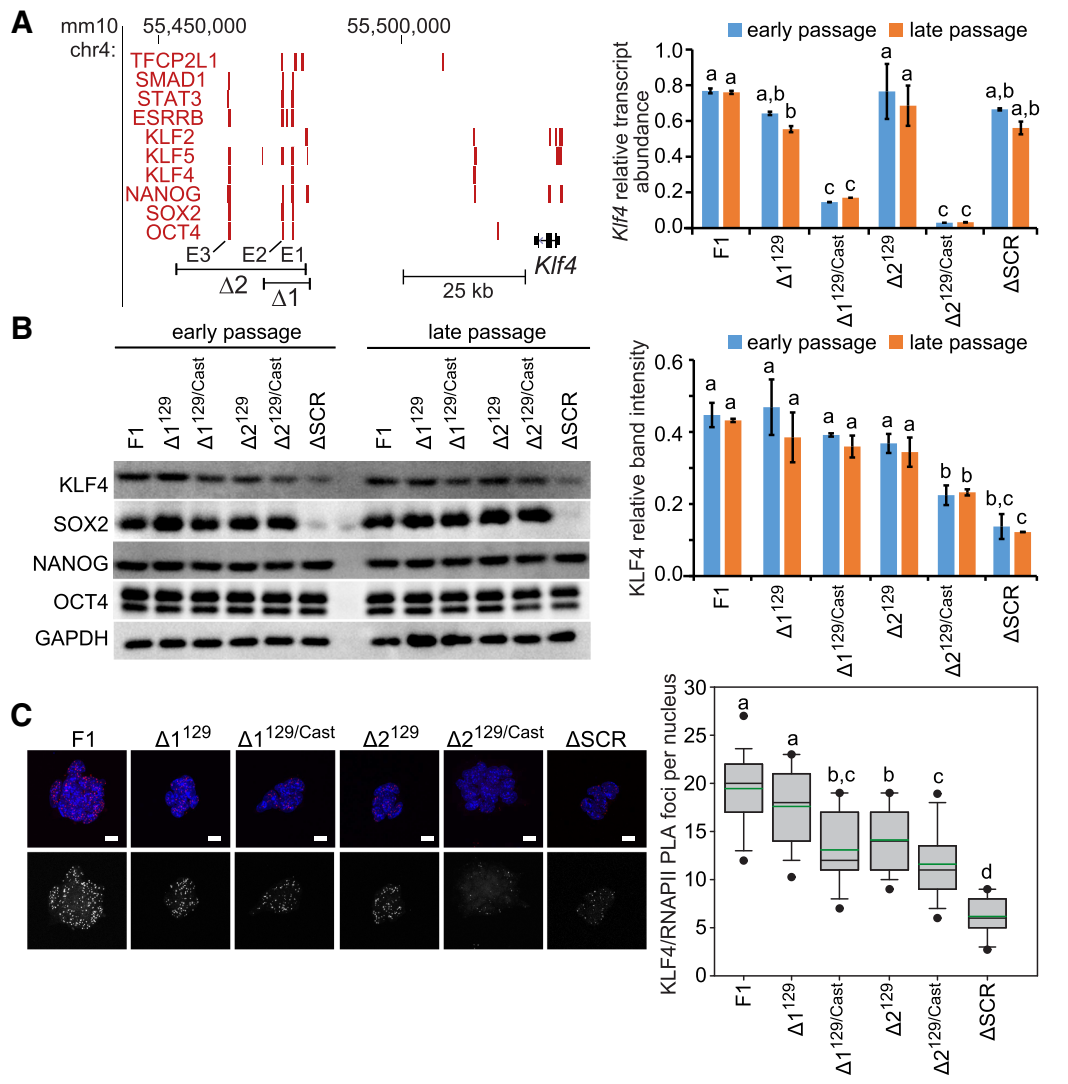

Figure 1. Klf4 transcript and protein levels are uncoupled in ES cells maintained in LIF/2i. $(A$, left $)$ Genome browser view of enhancer regions (E1, $\mathrm{E} 2$, and E3) downstream from Klf4, indicating $\Delta 1$ and $\Delta 2$ deletions. Red bars show binding of transcription factors involved in pluripotency. Data are displayed on the $\mathrm{mm} 10$ assembly of the University of California at Santa Cruz (UCSC) Genome Browser. (Right) Total Klf4 transcript levels quantified relative to Gapdh from three biological replicates of both early and late passages of Klf4 enhancer-deleted clones and the $\Delta \mathrm{SCR}^{129 / \text { Cast }}$ clone $(\triangle \mathrm{SCR})$. Error bars represent standard deviation; statistical differences determined by twoway ANOVA $(P<0.05)$ are indicated by different letters. $(B$, left $)$ Immunoblots showing the levels of pluripotency transcription factors in both early and late passages for Klf4 enhancer-deleted clones. GAPDH levels indicate sample loading. (Right) KLF4 quantified relative to GAPDH from three biological replicates of early and late passages for Klf4 enhancer-deleted clones and the $\Delta \mathrm{SCR}^{129 / \text { Cast }}$ clone $(\triangle \mathrm{SCR})$. Error bars represent standard deviation; statistical differences determined by twoway ANOVA $(P<0.05)$ are indicated by different letters. $(C)$ Proximity ligation amplification (PLA) displays the interaction between KLF4 and RNA polymerase II (RNAPII) in Klf4 enhancer-deleted and $\Delta \mathrm{SCR}^{129 / \text { Cast }}$ clones $(\Delta \mathrm{SCR})$. Images shown are maximum intensity projections. Merged images at the top display DAPI in blue and PLA in red; PLA signal is shown in grayscale at the bottom. Scale bar, $10 \mu \mathrm{m}$. (Right) Box and whisker plots indicate the number of PLA foci per nucleus. Boxes indicate interquartile range of intensity values, and whiskers indicate the 10th and 90th percentiles; outliers are shown as black dots. Images were collected from at least three biological replicates, and $\geq 100$ nuclei were quantified for each clone. Statistical differences determined by one-way ANOVA $(P<0.05)$ are indicated by different letters.

stable as KLF4 with $t_{1 / 2} \sim 3 \mathrm{~h}$, and their stability is not affected by differentiation (Supplemental Fig. S2). KLF4 protein stability is not affected by deletion of its downstream enhancers, explaining the modest reduction in KLF4 protein in these clones (Supplemental Fig. S2).

Reduced KLF4 protein stability upon differentiation by removal of LIF/2i suggested that extrinsic factors that activate signaling cascades could be involved in regulating KLF4 protein stability. Upon investigating the effect of individual signaling pathways on KLF4 stability, we observed that activation of the MAPK pathway, which occurs after MEKi removal, has the most significant effect on KLF4 stability, followed by inhibition of the JAKSTAT pathway by LIF removal (Fig. 2B). Removing GSK3i and allowing activation of Wnt signaling for $24 \mathrm{~h}$ did not have a significant effect on KLF4 stability. For ES cells maintained in LIF/serum, KLF4 displayed low protein stability similar to the observed stability after removal of MEKi (Supplemental Fig. S2). Previous studies demonstrated that these signaling pathways regulate gene transcription (Nichols and Smith 2009; Zhang et al. 2010; Theunissen et al. 2011; Dhaliwal et al. 2018); therefore, we investigated the effect of individual signaling pathways on Klf4, Nanog, Oct4, Sox2, Klf2, and Klf5 transcription. We observed that the removal of MEKi alone or in combination with LIF/GSK3i for $12 \mathrm{~h}$ significantly reduces Klf4 and Nanog transcription, but Oct4, Sox2, Klf2, and Klf5 transcription remains unaffected (Supplemental Fig. S3). As changes to Klf4 transcription could confound the analysis of KLF4 stability during differentiation, we investigated the stability of KLF4-GFP controlled by a CMV promoter upon removal of MEKi, LIF, or GSK3i (Supplemental Fig. S4). The stability of KLF4GFP in these conditions was similar to the stability of the endogenous protein, with removal of MEKi or LIF reducing KLF4 stability more dramatically than removal of GSK3i.

The MAPK pathway has been shown to have a role in KLF4 nuclear localization, ubiquitination, and degradation (Kim et al. 2012, 2014; Dhaliwal et al. 2018). As early as $6 \mathrm{~h}$ after MEKi removal, KLF4 was observed to exit the nucleus, and cytoplasmic KLF4 undergoes proteasomal degradation (Dhaliwal et al. 2018). In addition, nuclear export was found to depend on the presence of both a KLF4 nuclear export signal (NES1 at 97-107) and the ERK phosphorylation site in KLF4 at S132 (Dhaliwal et al. 2018). KLF4 nuclear export after MEKi removal, which allows ERK activation, could explain the observed reduction in KLF4 protein stability; to investigate this further, we used ES cells with stable integration of wild-type (WT) 

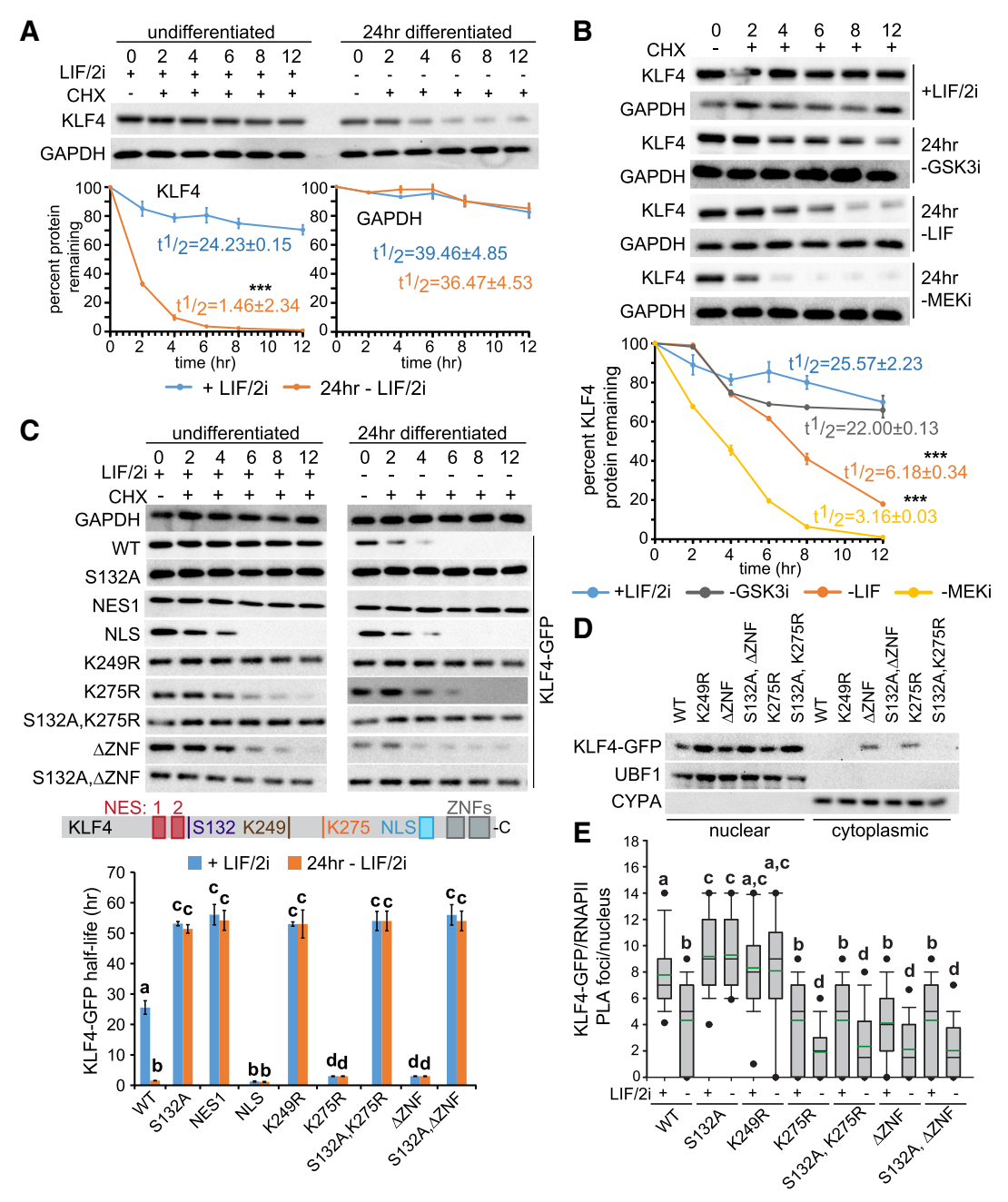

Figure 2. KLF4 protein stability is regulated by the LIF and MAPK signaling pathways, nuclear anchoring, and posttranslational modifications to KLF4. (A) Immunoblots for KLF4 and GAPDH in ES cells cultured with LIF/2i (undifferentiated) and $24 \mathrm{~h}$ after removal of LIF/2i, sampled at $0,2,4,6,8$, and $12 \mathrm{~h}$ after cycloheximide $(\mathrm{CHX})$ treatment. GAPDH levels were used as a control and displayed the expected half-life $\left(t_{1 / 2}>30 \mathrm{~h}\right)$. (Bottom) The percentage of remaining KLF4 or GAPDH protein was calculated from the intensity of CHX treatment immunoblots, measured in three biological replicates. KLF4 or GAPDH protein half-life in the indicated clones maintained in the presence or absence of LIF/2i was calculated for each time series replicate by best fit to exponential decay. Error bars represent standard deviation of three biological replicates. Statistical differences were determined by two-tailed $t$-test. $\left({ }^{* * *}\right) P<$ 0.001. (B) Immunoblots for KLF4 and GAPDH in ES cells cultured with LIF/ $2 \mathrm{i}$ and $24 \mathrm{~h}$ after removal of individual medium components (GSK3i, LIF, or MEKi), sampled at 0, 2, 4, 6, 8, and $12 \mathrm{~h}$ after CHX treatment. (Bottom) The percentage of remaining KLF4 protein was calculated from the intensity of CHX treatment immunoblots, measured in three biological replicates. Half-life was calculated for each time series replicate by best fit to exponential decay. The error bars represent standard deviation. Statistical differences between protein half-life in different culture conditions compared with $\mathrm{LIF} / 2 \mathrm{i}$ were determined by two-tailed $t$-test. $(* * *) P<0.001$. (C) Immunoblots for wild-type (WT) KLF4-GFP, the indicated mutants, and GAPDH from ES cells cultured with $\mathrm{LIF} / 2 \mathrm{i}$ and $24 \mathrm{~h}$ after LIF/2i removal, sampled at 0,2 , 4, 6, 8, and $12 \mathrm{~h}$ after CHX treatment. (Middle) A schematic of mouse KLF4 is shown depicting nuclear export signals (NESs), ERK phosphorylation site S132, ubiquitination site K249, sumoylation site K275, nuclear localization signal (NLS), and zinc fingers (ZNF). (Bottom) The calculated KLF4 protein half-life is shown in the indicated mutants cultured with LIF $/ 2 \mathrm{i}$ and $24 \mathrm{~h}$ after LIF $/ 2 \mathrm{i}$ removal. Half-life was calculated for each time series replicate by best fit to exponential decay. Error bars represent standard deviation of three biological replicates. Statistical differences determined by two-way ANOVA $(P<0.05)$ are indicated by different letters. $(D)$ Nuclear and cytoplasmic fractions prepared from WT KLF4-GFP and the indicated mutants (immunoblots probed with anti-GFP) indicated the expression and localization of KLF4-GFP. UBF1 and CYPA were used to analyze the purity of nuclear and cytoplasmic fractions, respectively. (E) Box and whisker plots display the number of KLF4GFP/RNAPII PLA foci per nucleus for WT and the indicated mutants. Boxes indicate interquartile range of intensity values, and whiskers indicate the 10th and 90th percentiles; outliers are shown as black dots. Images were collected from at least three biological replicates, and $\geq 100$ nuclei were quantified for each sample. Statistical differences determined by two-way ANOVA $(P<0.05)$ are indicated by different letters.

KLF4-GFP, the NES1 mutant [KLF4(NES1)-GFP], KLF4 (S132A)-GFP, and a nuclear localization signal (NLS) mutant [KLF4(NLS)-GFP]. WT KLF4-GFP displayed stability similar to endogenous KLF4 with a $t_{1 / 2}>24 \mathrm{~h}$ in undifferentiated cells that was reduced to $<2 \mathrm{~h}$ after $24 \mathrm{~h}$ of differentiation (Fig. 2C). Both of the constitutively nuclear mutants-KLF4(NES1)-GFP and KLF4(S132A)GFP-were highly stable proteins $(t>51 \mathrm{~h})$, and this stability was not affected by differentiation. In contrast, the constitutively cytoplasmic KLF4(NLS)-GFP was unstable, with a $t_{1 / 2}<2 \mathrm{~h}$ in both undifferentiated and differentiated cells. Together, these data indicate that nuclear localization is critical for KLF4 protein stability.

\section{KLF4 association with DNA and RNAPII is required to maintain protein stability through nuclear anchoring}

As localization to the nucleus increased the stability of KLF4, we next investigated the protein stability of mutants with disrupted KLF4 transcription factor function. KLF4 contains two C-terminal zinc finger (ZNF) domains required for DNA binding (Wei et al. 2009; Schuetz et al. 2011) and a sumoylation site at K275 shown to be important for transactivation of target promoters in reporter assays (Du et al. 2010). The amino acids surrounding K275 are highly conserved, highlighting conservation of the KLF4 sumoylation site (Supplemental Fig. S5). Stable 
expression of KLF4 loss-of-function mutants with a mutated sumoylation site [KLF4(K275R)-GFP] or deleted zinc fingers (KLF4 $\Delta$ ZNF-GFP) disrupted their nuclear localization (Fig. 2D) and reduced their protein stability $\left(\mathrm{t}_{1 / 2}\right.$ of $\left.\sim 3 \mathrm{~h}\right)$ when compared with WT KLF4-GFP (Fig. 2C). Mutation of S132, together with K275 or zinc finger deletion, restored nuclear anchoring and increased the stability of KLF4 (Fig. 2C,D). Interestingly, disruption of either KLF4 sumoylation (K275R) or DNA binding $(\triangle \mathrm{ZNF})$ interfered with recruitment to RNAPII-S5P nuclear compartments, as shown by the decrease in the number of KLF4/RNAPII PLA foci per nucleus compared with WT KLF4-GFP (Fig. 2E). Introducing the S132 mutation into the KLF4 loss-of-function mutants restored nuclear anchoring but did not restore RNAPII association, indicating that sumoylation and the DNA-binding domains are required for KLF4 association with RNAPII.

\section{Ubiquitination of KLF4 is required for nuclear export and degradation during the course of differentiation}

Upon phosphorylation by ERK and subsequent nuclear export, KLF4 has been shown to be degraded, causing ES cell differentiation (Kim et al. 2012; Dhaliwal et al. 2018). As expected, treatment with the proteasome inhibitor (MG132) prevented the decrease in the levels of KLF4 protein normally observed in cells differentiated for $24 \mathrm{~h}$ (Fig. 3A). A previous study showed that K232 was the most critical residue in human KLF4 for ubiquitination and degradation (Lim et al. 2014). KLF4 is highly conserved in this region, with $\mathrm{K} 249$ in the mouse sequence predicted as a ubiquitination site by UbPred and NetChop (Supplemental Fig. S5; Keşmir et al. 2002; Radivojac et al. 2010). In order to further investigate the role of K249 in KLF4 function, we generated stable ES lines expressing a KLF4 ubiquitination site mutant, KLF4 (K249R)-GFP. KLF4(K249R)-GFP is nuclear in undifferentiated ES cells, similar to the WT protein (Fig. 2D), but displayed an increased $t_{1 / 2}$ of $53 \mathrm{~h}$ independent of culture conditions similar to the S132 and NES1 mutants (Fig. $2 \mathrm{C}$ ), indicating that blocking KLF4 ubiquitination prevents the loss of KLF4 stability upon differentiation. Blocking KLF4 ubiquitination by mutation of K249 does not disrupt interaction with RNAPII-S5P in undifferentiated cells and prevents the loss of KLF4/RNAPII-S5P interaction associated with differentiation (Fig. 2E). As this was similar to what occurs in the KLF4 S132 and NES1 mutants, which showed disrupted nuclear export and no interaction with Xportin 1 (XPO1) (Dhaliwal et al. 2018), we investigated the interaction between KLF4(K249R)-GFP and XPO1 in differentiating ES cells by PLA. Indeed, mutation of K249 did disrupt the interaction between KLF4 and XPO1 normally observed in differentiating cells (Fig. 3B).

We next investigated the ubiquitination status of KLF4 in nuclear and cytoplasmic fractions and the effect of K249 mutation on KLF4 ubiquitination (Fig. 3C). KLF4-GFP protein has a predicted molecular weight of $81 \mathrm{kDa}$; in ES cells, immunoblot for KLF4-GFP protein identifies a prominent band at $84 \mathrm{kDa}$ (Fig. 3C, input).

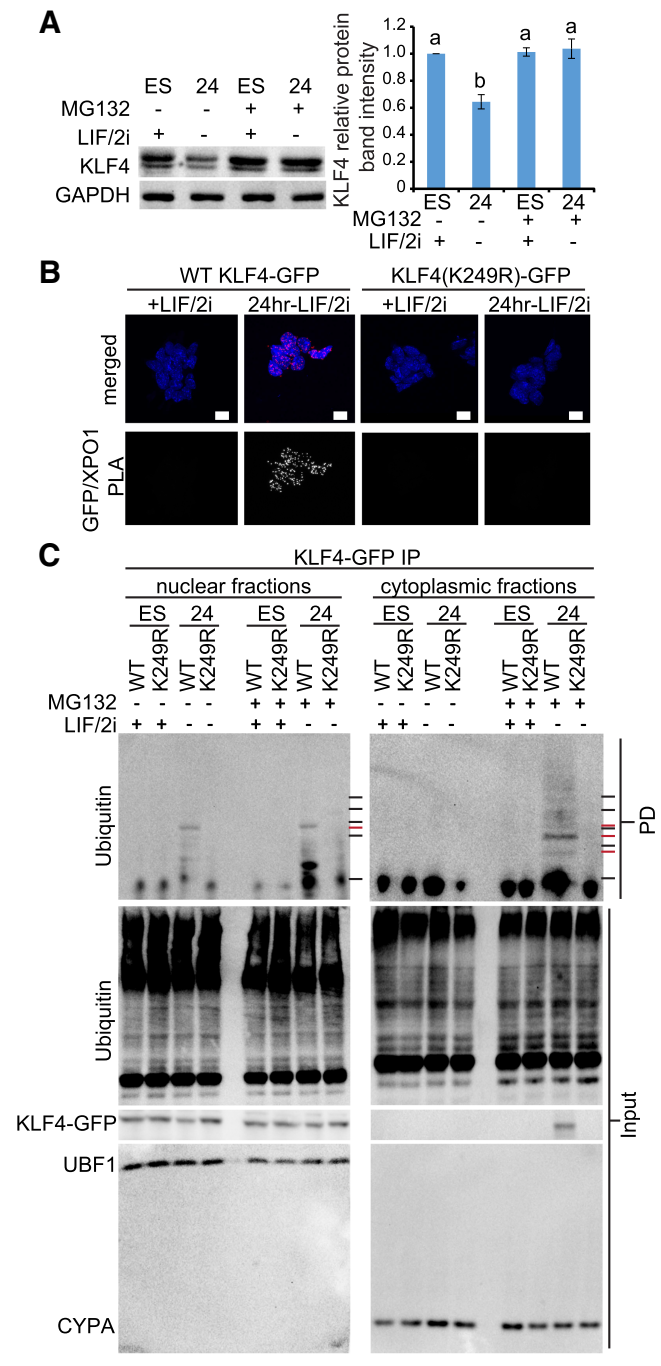

Figure 3. Ubiquitination of KLF4 is required for nuclear export and degradation during differentiation. $(A)$ Immunoblots for KLF4 in whole-cell lysate prepared from ES cells cultured with LIF/ $2 \mathrm{i}$ and $24 \mathrm{~h}$ after removal of LIF/2i, treated or not with proteasome inhibitor MG132. GAPDH levels indicate sample loading. (Right) Quantification of KLF4 protein intensity level relative to GAPDH and ES cell levels (+LIF/2i, -MG132) in three biological replicates. Error bars represent standard deviation. Statistical differences determined by one-way ANOVA $(P<0.05)$ are displayed by different letters. $(B)$ PLA displays the interaction between KLF4-GFP/XPO1 in WT and K249R mutant-expressing cells cultured with LIF/2i and $24 \mathrm{~h}$ after removal of LIF/2i. Images shown are maximum intensity projections. Merged images display DAPI in blue and PLA in red. Scale bar, $10 \mu \mathrm{m}$. (C) KLF4-GFP immunoprecipitation (IP) using anti-GFP from nuclear and cytoplasmic fractions of WT and the K249R mutant-expressing cells cultured with $L I F / 2 \mathrm{i}$ and $24 \mathrm{~h}$ after removal of $\mathrm{LIF} / 2 \mathrm{i}$, treated and untreated with proteasomal inhibitor MG132. Input and pull-down (PD) samples were probed with antiubiquitin. At the right of the ubiquitin blot, molecular weight markers are indicated by black lines (from the top: 250, 150, 100, 75, $50 \mathrm{kDa}$ ). The red lines indicate bands with calculated molecular weights of $95 \mathrm{kDa}$ in the nuclear fraction and 105,84 , and $72 \mathrm{kDa}$ in the cytoplasmic fraction. Cyclophilin A (CYPA) and the nucleolar protein upstream binding factor (UBF1) were detected simultaneously for all samples and reveal purity of the cytoplasmic and nuclear fractions, respectively. 
WT KLF4-GFP immunoprecipitated from the nuclear fraction using an anti-GFP antibody displayed a prominent ubiquitin band at $95 \mathrm{kDa}$ (Fig. 3C), which could correspond to monoubiquitination of KLF4-GFP, as ubiquitin has a molecular weight of $8.5 \mathrm{kDa}$. This band was apparent only after removal of $\mathrm{LIF} / 2 \mathrm{i}$ and was absent from the KLF4(K249R)-GFP mutant, revealing that ubiquitination depends on the presence of K249. WT KLF4-GFP immunoprecipitated from cytoplasmic fractions using an anti-GFP antibody revealed more of a high-molecular-weight laddering pattern above $105 \mathrm{kDa}$ that could correspond to polyubiquitination of cytoplasmic KLF4-GFP in MG132-treated differentiated $(-\mathrm{LIF} / 2 \mathrm{i})$ cells. The presence of bands below $95 \mathrm{kDa}$ could represent partial KLF4-GFP degradation (Fig. 3C). These ubiquitin immunoreactive bands were not detected in the absence of MG132, suggesting that cytoplasmic polyubiquitinated KLF4 is rapidly degraded. KLF4(K249R)-GFP was not detected in the cytoplasmic fraction by either ubiquitin or GFP antibodies, indicating that mutation of the ubiquitination site blocked nuclear export of KLF4-GFP.

The loss of KLF4 protein stability is required for ES cell differentiation

To evaluate the role of KLF4 stability in pluripotency maintenance and exit from the pluripotent state, we differentiated cells expressing WT KLF4-GFP or mutant proteins for $5 \mathrm{~d}$ in the absence of LIF/2i. Cells in the pluripotent state exhibit high alkaline phosphatase activity, which is lost upon differentiation. Cells that express KLF4 mutants with increased protein stability [KLF4 (K249R)-GFP and KLF4(S132A)-GFP] maintain alkaline phosphatase activity $5 \mathrm{~d}$ after removal of $\mathrm{LIF} / 2 \mathrm{i}$, indicating a block in differentiation (Fig. 4A). In addition, expression of either KLF4(K249R)-GFP or KLF4(S132A)-GFP prevented decreased expression of endogenous pluripotency transcription factors normally down-regulated during differentiation after removal of LIF/2i (Fig. 4B).

To further investigate the importance of the role of KLF4 as a transcription factor in this context, we evaluated the role of the sumoylation site that we found was important for recruitment to the RNAPII compartment and which has been shown to be involved in transactivation ( $\mathrm{Du}$ et al. 2010). Although expression of the K275 sumoylation site mutant does not affect differentiation, mutation of this site in an S132A ERK phosphorylation site mutant background abolished the ability of the compound mutant to maintain naïve pluripotency (Fig. 4). Similarly, we found that KLF4 requires the ability to bind DNA through its zinc finger domains, as differentiation occurred in cells expressing the compound mutant KLF4(S132A $\triangle \mathrm{ZNF})-\mathrm{GFP}$, indicating that this mutant was unable to maintain pluripotency in the absence of LIF/2i. Together, these data indicate that maintaining high KLF4 protein stability blocks pluripotency exit and that KLF4 requires the ability to bind DNA and associate with RNAPII to maintain the pluripotent state.
A

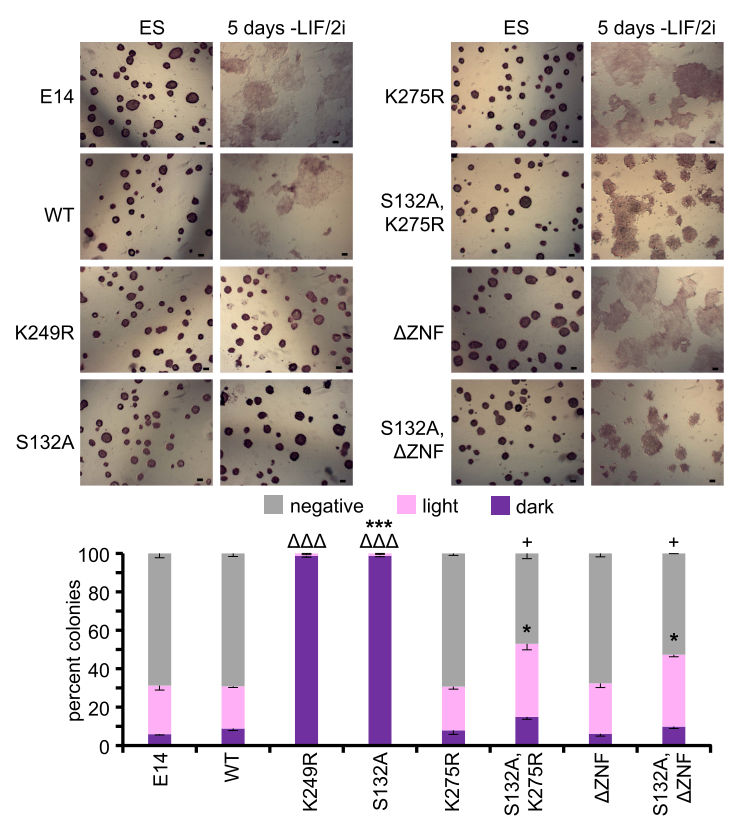

B E14 $\square$ WT K249R S132A K275R $\square$ S132A,K275R $\triangle$ ZNF $\square$ S132A, $\Delta$ ZNF

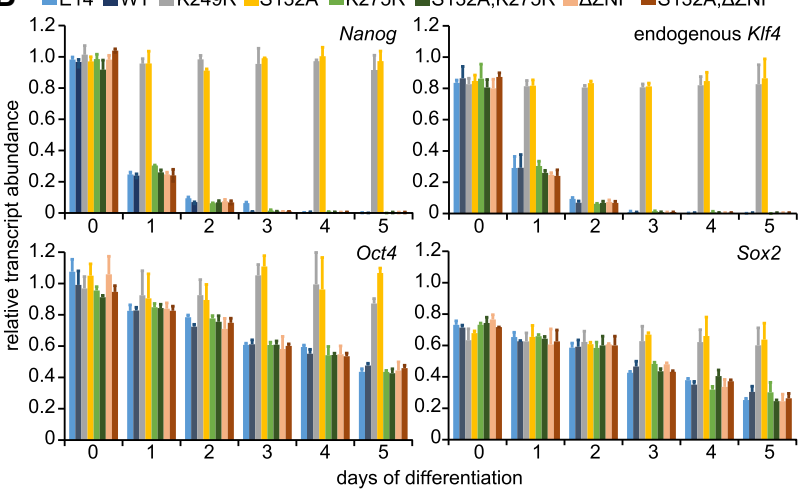

Figure 4. Loss of KLF4 protein stability is required for ES cell differentiation. (A) Alkaline phosphatase staining of untransfected E14TG2a (E14) ES cells, WT, and the indicated KLF4-GFP mutant-expressing cells in undifferentiated ES cells and $5 \mathrm{~d}$ after $\mathrm{LIF} / 2 \mathrm{i}$ removal. Scale bar, $50 \mu \mathrm{m}$. After $5 \mathrm{~d}$ of LIF/2i removal, positive and negative colonies were counted from at least three replicates for each indicated mutant, revealing that expression of WT KLF4-GFP does not block differentiation, whereas expression of the S132A or K249R mutant does. Error bars represent standard deviation. Statistical differences between each type of colony compared with WT KLF4-GFP were determined by $t$-test. $(\Delta \Delta \Delta)$ $P<0.001$ for dark colonies; $\left({ }^{*}\right) P<0.5$ for light colonies; $\left({ }^{* * *}\right) P<$ 0.001 for light colonies; $(+) P<0.5$ for negative colonies. $(B)$ Transcripts for endogenous Klf4, Nanog, Oct4, and Sox2 were quantified relative to Gapdh levels in three biological replicates of untransfected E14 ES cells, WT cells, and the indicated KLF4GFP mutant cells, revealing that only KLF4(S132A)-GFP and KLF4(K249R)-GFP blocked differentiation. Error bars represent standard deviation.

Phosphorylated STAT3 (pSTAT3) stabilizes KLF4 protein in nuclear complexes

Removal of LIF from cells maintained in LIF/2i significantly reduced KLF4 protein stability from $t_{1 / 2}=26 \mathrm{~h}$ to 
$t_{1 / 2}=6 \mathrm{~h}$ after $24 \mathrm{~h}$ (Fig. 2B). LIF signaling activates the JAK-STAT pathway in ES cells, causing phosphorylation and activation of STAT3 (Raz et al. 1999; Zhang et al. 2010; Hirai et al. 2011; Ohtsuka et al. 2015). After removal of LIF/2i, the levels of pSTAT3 in the nucleus decrease rapidly and are undetectable after $6 \mathrm{~h}$ of differentiation (Supplemental Fig. S6). PLA for STAT3/KLF4 during early differentiation showed that interaction between these two proteins occurs in undifferentiated cells but is lost during the first $24 \mathrm{~h}$ of differentiation (Supplemental Fig. S6). We next examined whether a short 1-h LIF induction in cells differentiated for $24 \mathrm{~h}$ could restore STAT3/KLF4 interaction and KLF4 protein stability. The $t_{1 / 2}$ of KLF4 increased from $<2 \mathrm{~h}$ to $24 \mathrm{~h}$ after a 1-h treatment with LIF (Fig. 5A). This short LIF treatment also increased the levels of Klf4 transcript and protein in 24-h differentiated cells (Fig. 4D,E) and restored the interaction between KLF4 and STAT3 (Fig. 5B-D). As LIF regulated KLF4 at both the transcriptional and posttranscriptional levels, we repeated the 1-h LIF treatment in cells treated at the same time with the protein synthesis inhibitor cycloheximide (CHX). Even in the presence of CHX, which inhibits de novo protein synthesis, an increase in KLF4 stability was observed after a 1-h LIF treatment (Supplemental Fig. S7). Next, we investigated KLF4 association with RNAPII by PLA, which revealed a significant increase in the number of interaction foci per nucleus after the 1-h LIF induction (Fig. 5E). These data indicate that pSTAT3 stabilizes KLF4 protein in ES cells and is involved in recruiting KLF4 to the RNAPII compartment.

\section{SOX2 and NANOG regulate KLF4 posttranslationally by stabilizing KLF4 protein in nuclear complexes}

As association with pSTAT3 increased the stability of KLF4 protein after a 1-h treatment with LIF, we next investigated whether additional pluripotency transcription factors (SOX2 and NANOG) had a role in controlling KLF4 stability. Cells with compromised SOX2 protein levels due to homozygous deletion of the SCR express reduced levels of KLF4 protein but not RNA (Fig. 1), which could be due to reduced KLF4 protein stability in these cells. The $\Delta \mathrm{SCR}^{129 / \text { Cast }}$ cells are female F1 ES cells that are partially differentiated; they have one inactivated $\mathrm{X}$ chromosome and display up-regulation of genes normally expressed in trophoblast stem cells, determined by RNA sequencing (RNA-seq) (Zhou et al. 2014). In addition, we showed that the levels of Oct4, Nanog, Klf2, and Klf5 transcripts as well as protein were not affected by deletion of the SCR (Supplemental Fig. S1).

Evaluating KLF4 protein stability in $\triangle \mathrm{SCR}^{129 / \text { Cast }}$ cells revealed that KLF4 is unstable with a $t_{1 / 2}<2 \mathrm{~h}$, and this reduced stability is unaffected by removal of LIF/2i (Fig. 6A). To evaluate the role of SOX2 in stabilizing KLF4 protein, $\Delta \mathrm{SCR}^{129 / \text { Cast }}$ cells were transfected with Sox2-t2A-GFP. Transfection restored Sox2 mRNA and protein to WT levels and significantly increased KLF4 protein but not transcript levels, indicating that regulation of KLF4 occurs posttranscriptionally by SOX2 (Fig. 6B,C). In addition, the stability of KLF4 protein increased significantly from
A
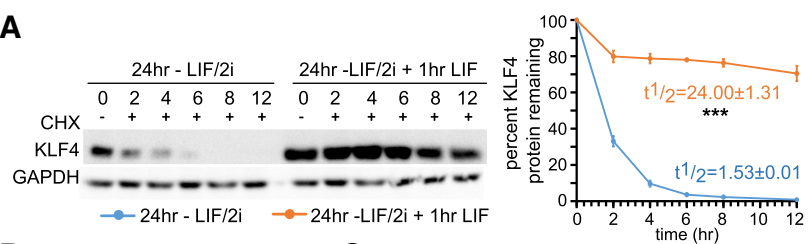

B

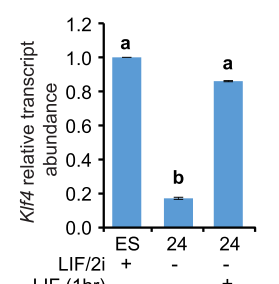

C

D
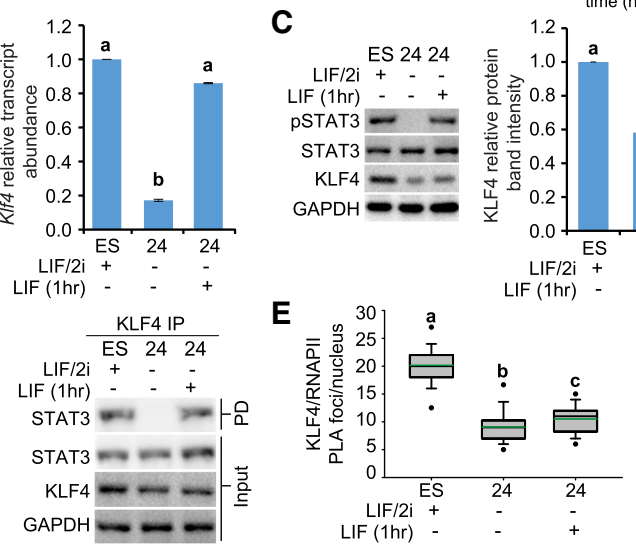

Figure 5. pSTAT3 stabilizes KLF4 protein in nuclear complexes. (A) Immunoblots for KLF4 and GAPDH in ES cells $24 \mathrm{~h}$ after LIF/ 2i removal and $24 \mathrm{~h}$ after LIF/2i removal followed by a 1-h pretreatment with LIF, sampled at $0,2,4,6,8$, and $12 \mathrm{~h}$ after CHX treatment. GAPDH levels were used as a control and displayed the expected half-life $\left(t_{1 / 2}>30 \mathrm{~h}\right)$. (Right) The percentage of remaining KLF4 was calculated from the intensity of CHX treatment immunoblots, measured in three biological replicates. Half-life was calculated for each time series replicate by best fit to exponential decay. Error bars represent standard deviation. Statistical differences between protein half-life were determined by two-tailed $t$-test. $\left.{ }^{* * *}\right) P<0.001$. (B) Transcript levels for Klf4 were quantified relative to Gapdh and ES levels in three biological replicates of ES cells cultured with LIF/2i, $24 \mathrm{~h}$ after LIF/2i removal, and $24 \mathrm{~h}$ after LIF/2i removal followed by a 1-h treatment with LIF. Error bars represent standard deviation. Statistical differences were determined by one-way ANOVA $(P<0.05)$ and are displayed by different letters. $(C)$ Immunoblots for pSTAT3 (Tyr705) and total STAT3 in ES cells cultured with LIF/2i, $24 \mathrm{~h}$ after LIF/2i removal and $24 \mathrm{~h}$ after LIF/2i removal followed by a $1-\mathrm{h}$ treatment with LIF. GAPDH levels indicate sample loading. (Right) KLF4 protein relative to GAPDH and ES levels in three biological replicates of ES cells cultured with LIF/2i, $24 \mathrm{~h}$ after LIF/ $2 \mathrm{i}$ removal, and $24 \mathrm{~h}$ after LIF/2i removal followed by a 1-h treatment with LIF. Error bars represent standard deviation. Statistical differences determined with one-way ANOVA $(P<0.05)$ are displayed by different letters. $(D)$ KLF4 immunoprecipitation (IP) in ES cells cultured with LIF/2i, $24 \mathrm{~h}$ after LIF/2i removal, and $24 \mathrm{~h}$ after LIF/2i removal followed by a 1-h treatment with LIF, probed with anti-STAT3. (PD) Pull-down. GAPDH levels indicate sample loading of the input. (E) Box and whisker plots display the number of KLF4/RNAPII PLA foci per nucleus for ES cells cultured with LIF/2i, $24 \mathrm{~h}$ after LIF/2i removal, and $24 \mathrm{~h}$ after LIF/ $2 \mathrm{i}$ removal followed by a 1 -h treatment with LIF. Boxes indicate interquartile range of intensity values, and whiskers indicate the 10th and 90th percentiles; outliers are shown as black dots. Images were collected from at least three biological replicates, and $\geq 100$ nuclei were quantified for each sample. Statistical differences determined with one-way ANOVA $(P<0.05)$ are indicated by different letters. 


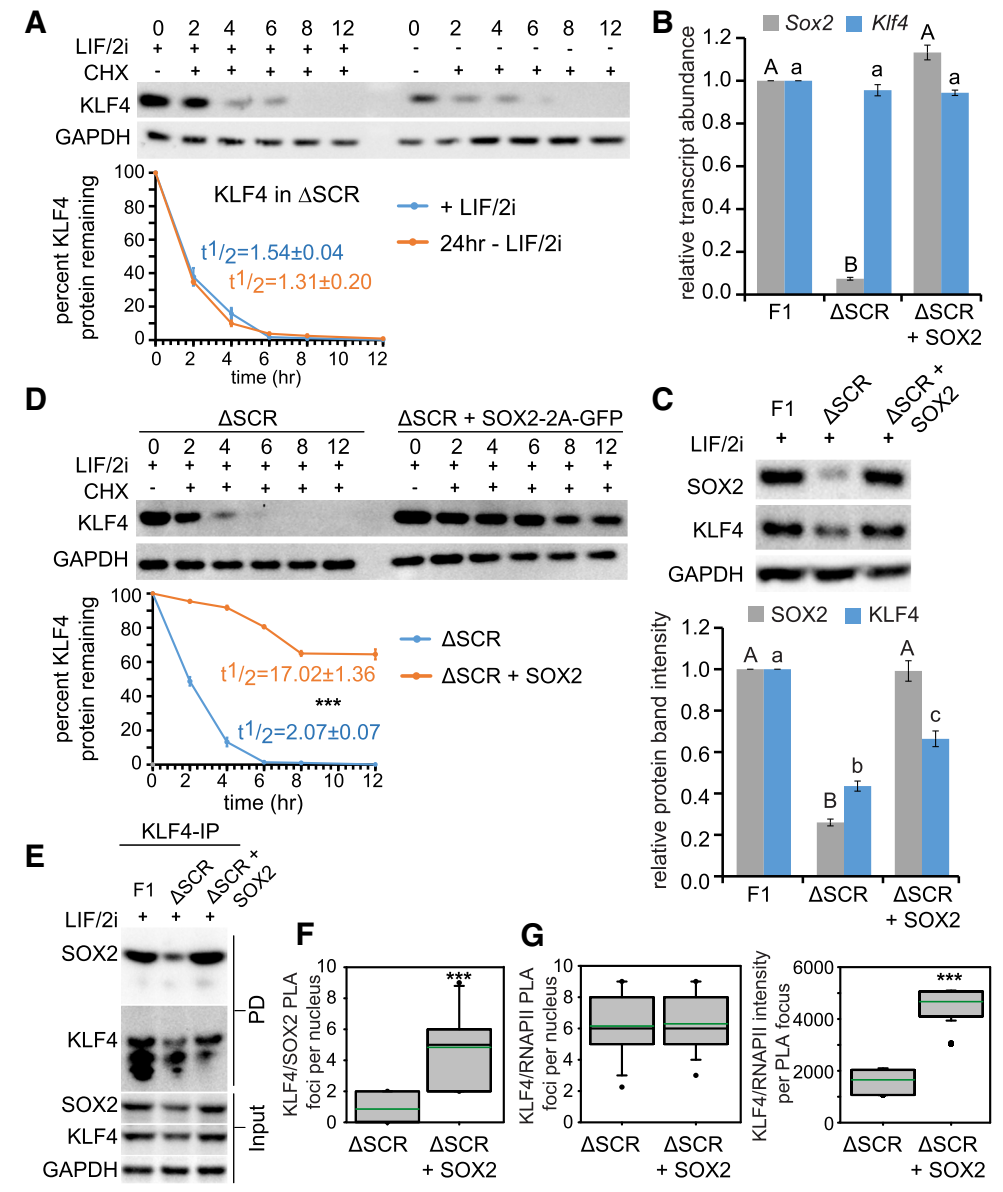

Figure 6. SOX2 stabilizes KLF4 protein in nuclear complexes. $(A)$ Immunoblots for KLF4 in the $\triangle S_{C} \mathrm{R}^{129 / \text { Cast }}$ clone $(\triangle \mathrm{SCR})$, cultured with $\mathrm{LIF} / 2 \mathrm{i}$ and $24 \mathrm{~h}$ after removal of LIF/2i, sampled at $0,2,4,6,8$, and $12 \mathrm{~h}$ after CHX treatment. GAPDH levels were used as a control and displayed the expected half-life $\left(t_{1 / 2}>30 \mathrm{~h}\right)$. The percentage of remaining KLF4 protein was calculated from the intensity of CHX treatment immunoblots, measured in three biological replicates. KLF4 protein half-life in the presence or absence of LIF/ $2 \mathrm{i}$ was calculated for each time series replicate by best fit to exponential decay. Error bars represent standard deviation of three biological replicates. Two-tailed $t$-test revealed that the difference between these two conditions was not statistically significant. (B) Sox2 and Klf4 transcripts were quantified relative to Gapdh and F1 in three biological replicates of F1, $\triangle \mathrm{SCR}^{129 / \text { Cast }}$, and SOX2-2A-GFP transfected $\triangle \mathrm{SCR}^{129 / \text { Cast }}$. Error bars represent standard deviation. Statistical differences for each transcript were determined by one-way ANOVA $(P<0.05)$ and are displayed as uppercase letters for Sox2 and lowercase letters for Klf4. (C) Immunoblots for SOX2 and KLF4 in F1, $\triangle \mathrm{SCR}^{129 / \text { Cast }}$, and SOX2-2A-GFP transfected $\Delta \mathrm{SCR}^{129 / \text { Cast }}$ cells in LIF/2i. GAPDH levels indicate sample loading. Quantification of KLF4 and SOX2 relative to GAPDH and F1 in three biological replicates. Error bars represent standard deviation. Statistical differences for each protein were determined by one-way ANOVA $(P<$ $0.05)$ and are displayed as uppercase letters for SOX2 and lowercase letters for KLF4. (D) Immunoblot for KLF4 and GAPDH in untransfected and SOX2-2A-GFP transfected $\triangle S C R^{129 / \text { Cast }}$ cells in LIF/2i sampled at 0,2 , $4,6,8$, and $12 \mathrm{~h}$ after CHX treatment. GAPDH levels were used as a control and displayed the expected halflife $\left(t_{1 / 2}>30 \mathrm{~h}\right)$. The percentage of remaining KLF4 pro-

tein at $0,2,4,6,8$, and $12 \mathrm{~h}$ was calculated from the intensity of CHX treatment immunoblots, measured in three biological replicates. Half-life was calculated for each time series replicate by best fit to exponential decay. Error bars represent standard deviation. Statistical differences were determined by two-tailed $t$-test. $\left(^{* * *}\right) P<0.001$. (E) KLF4 immunoprecipitation (IP) from untransfected and SOX2-2AGFP transfected $\triangle S C R^{129 / C a s t} F 1$ ES cells probed with anti-SOX2 and anti-KLF4. (PD) Pull-down. GAPDH levels indicate loading of the input. $(F, G)$ Box and whisker plots. Boxes indicate interquartile range of intensity values, and whiskers indicate the 10th and 90 th percentiles; outliers are shown as black dots. Images were collected from at least three biological replicates, and $\geq 100$ nuclei were quantified for each sample. Statistical differences were determined by $t$-test. $\left(^{* * *}\right) P<0.001$. In $F$, the number of KLF4/SOX2 PLA foci per nucleus is shown. In $G$, the number of KLF4/RNAPII PLA foci per nucleus (left) and the total intensity value of each PLA focus (right) are shown.

$t_{1 / 2}=2 \mathrm{~h}$ to $t_{1 / 2}=17 \mathrm{~h}$ after SOX2 protein levels were restored (Fig. 6D). To investigate whether SOX2 could stabilize KLF4 through protein-protein interaction, KLF4 was immunoprecipitated to investigate its interaction with SOX2. Indeed, KLF4 and SOX2 do interact, and this interaction is restored in Sox2-t2A-GFP transfected cells (Fig. $6 \mathrm{E})$. Similarly, increased nuclear association between KLF4 and SOX2 was observed by PLA in Sox2-t2A-GFP transfected cells (Fig. 6F). Although the number of PLA foci per nucleus for KLF4 with RNAPII-S5P was unchanged when SOX2 protein levels were restored, the signal intensity per focus increased significantly, suggesting that SOX2 protein is involved in stabilizing KLF4 protein in nuclear transcriptional complexes (Fig. 6G).

As the levels of NANOG protein in the nucleus are drastically reduced by $24 \mathrm{~h}$ of differentiation, whereas SOX2 levels remain high at this time (Dhaliwal et al. 2018), we hypothesized that loss of NANOG protein, in addition to the loss of pSTAT3, could be involved in reduced
KLF4 stability after $24 \mathrm{~h}$ of differentiation. To test this hypothesis, cells differentiated for $24 \mathrm{~h}$ were transfected with Nanog-t2a-GFP to restore NANOG levels. After an additional $24 \mathrm{~h}$ in differentiation medium, KLF4 protein levels were restored in Nanog-t2a-GFP transfected cells without any change in Klf4 transcript levels, indicating a posttranscriptional regulatory mechanism (Fig. 7A,B). We also monitored the expression of Oct4, Sox2, Klf2, and Klf5 RNA and protein and found that they were not altered during this differentiation time frame or by Nanog-t2a-GFP transfection (Supplemental Fig. S8). Similar to what was observed for SOX2, NANOG expression in differentiated cells restores KLF4 protein stability from $t_{1 / 2}=1.5 \mathrm{~h}$ to $t_{1 / 2}=25 \mathrm{~h}$ (Fig. $7 \mathrm{C}$ ). To investigate whether NANOG could stabilize KLF4 through proteinprotein interaction, KLF4 was immunoprecipitated to investigate interaction with NANOG; indeed, KLF4 and NANOG do interact in differentiated cells transfected with Nanog-t2A-GFP (Fig. 7D). Restoring NANOG levels 


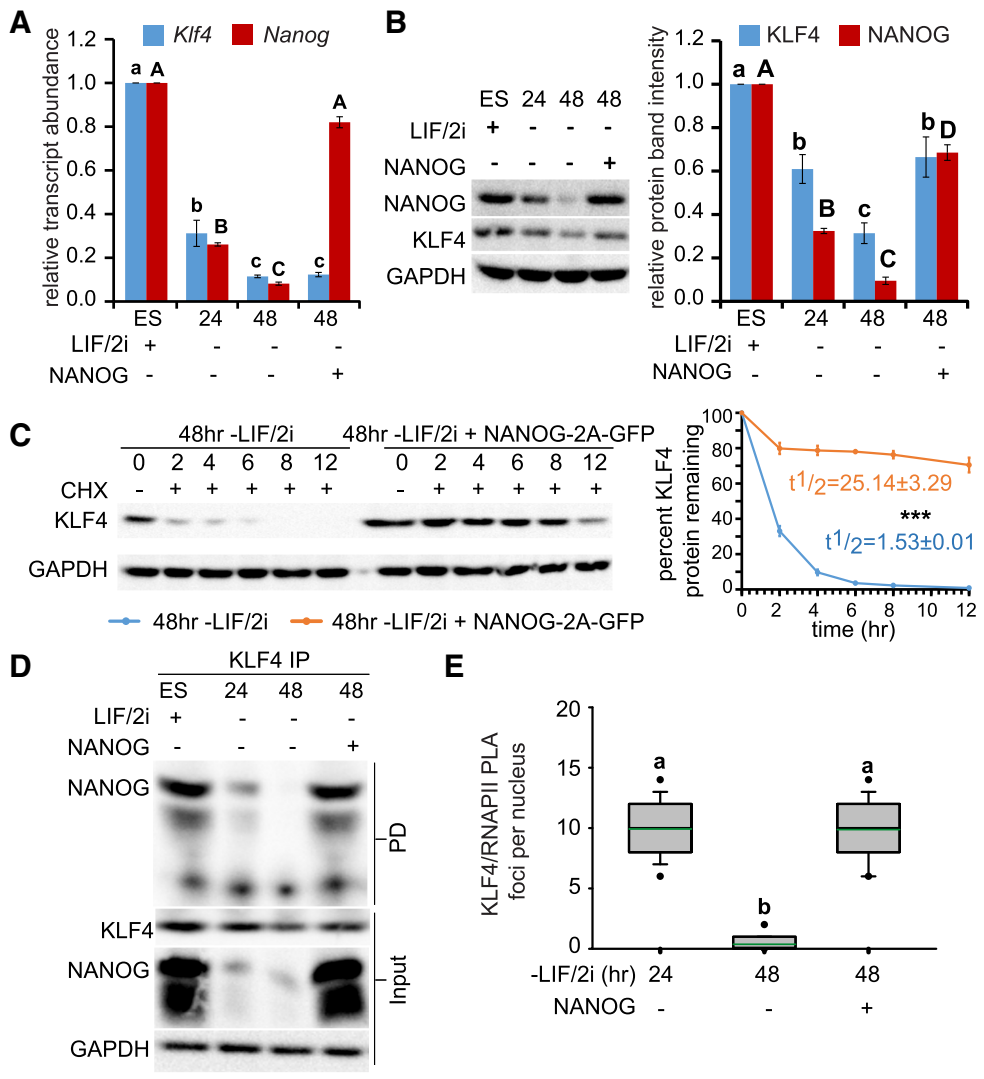

Figure 7. NANOG stabilizes KLF4 protein in nuclear complexes. (A) Transcript levels of Klf4 and Nanog were quantified relative to Gapdh and undifferentiated ES cells in three biological replicates of ES cells cultured with LIF/2i (ES), 24 and $48 \mathrm{~h}$ after removal of LIF/2i, and $48 \mathrm{~h}$ after removal of LIF/2i where NANOG-2A-GFP was transfected $24 \mathrm{~h}$ after removal of LIF/2i. Error bars represent standard deviation. Statistical differences for each transcript were determined by one-way ANOVA $\mid P<$ $0.05)$ and are displayed as lowercase letters for Klf4 and uppercase letters for Nanog. (B) Immunoblots for KLF4 and NANOG in ES cells cultured with LIF/2i, 24 and $48 \mathrm{~h}$ after removal of LIF/ $2 \mathrm{i}$, and $48 \mathrm{~h}$ after removal of LIF/2i where NANOG-2A-GFP was transfected $24 \mathrm{~h}$ after removal of LIF/2i. GAPDH levels indicate sample loading. (Right) Quantification of KLF4 and NANOG relative to GAPDH for three biological replicates is shown. Error bars represent standard deviation. Statistical differences for each protein were determined by oneway ANOVA $(P<0.05)$ and are displayed as lowercase letters for KLF4 and uppercase letters for NANOG. $(C)$ Immunoblot for KLF4 and GAPDH in ES cells $48 \mathrm{~h}$ after removal of LIF/2i and $48 \mathrm{~h}$ after removal of $\mathrm{LIF} / 2 \mathrm{i}$ where NANOG-2A-GFP was transfected $24 \mathrm{~h}$ after removal of LIF/2i, sampled at $0,2,4,6,8$, and $12 \mathrm{~h}$ after CHX treatment. GAPDH levels were used as a control and displayed the expected half-life $\left(t_{1 / 2}>30 \mathrm{~h}\right)$. (Right) The percentage of remaining KLF4 was calculated from the intensity of CHX treatment immunoblots, measured in three biological replicates. Half-life was calculated for each time series replicate by best fit to exponential decay. Error bars represent standard deviation. Statistical differences between protein half-life were determined by two-tailed $t$-test. $\left(^{* * *}\right) P<0.001$. (D) KLF4 immunoprecipitation (IP) from ES cells cultured with LIF/2i, 24 and $48 \mathrm{~h}$ after removal of $\mathrm{LIF} / 2 \mathrm{i}$, and $48 \mathrm{~h}$ after removal of LIF/2i where NANOG-2A-GFP was transfected $24 \mathrm{~h}$ after removal of LIF/2i, probed with anti-NANOG. (PD) Pull-down. GAPDH levels indicate loading of the input. (E) Box and whisker plots indicate the number PLA foci per nucleus for the interaction between KLF4 and RNAPII. Boxes indicate interquartile range of intensity values, and whiskers indicate the 10th and 90th percentiles; outliers are shown as black dots. Images were collected from at least three biological replicates, and $\geq 100$ nuclei were quantified for each sample. Statistical differences determined by one-way ANOVA $(P<$ 0.05 ) are indicated by different letters.

in differentiated cells not only increased the interaction between KLF4 and NANOG but also restored the interaction of KLF4 with RNAPII-S5P that is normally lost by 48 $h$ of differentiation, as revealed by PLA (Fig. 7E). Together, these data reveal that NANOG has a role in both stabilizing KLF4 protein and recruiting KLF4 to RNAPII compartments in the nucleus.

To investigate the role of pluripotency-associated transcription factors as stabilizing agents of KLF4 protein in another cellular context, we transfected HEK293 cells (which do not express these pluripotency regulators) with KLF4-GFP. KLF4-GFP displayed low protein stability in HEK293 cells with a $t_{1 / 2} \sim 1 \mathrm{~h}$ (Supplemental Fig. S8). Coexpression of SOX2 increased KLF4 protein stability; however, SOX2 was not able to increase the stability of KLF4 $\triangle$ ZNF-GFP, indicating that the DNA-binding zinc finger domains are important for stabilization of KLF4 by SOX2. This implicates protein-protein interaction between SOX2 and KLF4 as a key mechanism, as SOX2 has been shown to associate with KLF4 through the KLF4 zinc finger domains (Wei et al. 2009). In contrast, both WT KLF4-GFP and KLF4 $\triangle$ ZNF-GFP were stabilized to a similar extent by a complex of SOX2,
NANOG, and constitutively active STAT3 (CA-STAT3), and removal of SOX2 from this complex did not alter KLF4 stability (Supplemental Fig. S8). Addition of KLF2 to the full complex or cotransfection of only KLF2 with KLF4-GFP did not further stabilize KLF4 protein, indicating that there is no direct role for KLF2 in stabilizing KLF4 in this context.

Together, these data indicate that nuclear anchoring, through interaction with RNAPII complexes, pluripotency transcription factors, and association with DNA, maintains the stability of KLF4 protein in undifferentiated naïve pluripotent ES cells.

\section{Discussion}

The naïve pluripotent state of ES cells is regulated by pluripotency-associated transcription factors, which act in a complex interconnected network by binding their own regulatory elements as well as the regulatory elements of other genes throughout the genome to regulate transcription. Klf4 is regulated at a transcriptional level by downstream enhancers, which are required to maintain 
Klf4 transcript and protein levels in mouse ES cells maintained in LIF/serum (Xie et al. 2017). In contrast, we found that for ES cells maintained in LIF/2i, the same enhancers had a small role in maintaining KLF4 protein levels, as the high stability of the KLF4 protein in LIF/2i conditions buffered against the greatly reduced levels of Klf4 transcription in the absence of the enhancers. Removal of MEK inhibition greatly disrupted KLF4 stability, accounting for the dramatic differences between the requirements for Klf4 enhancers in LIF/serum compared with LIF/2i conditions. Furthermore, we found that KLF4 protein is stabilized both by domains within the protein that anchor KLF4 in the nucleus and by interaction with RNAPII, SOX2, NANOG, and pSTAT3. These findings detail a new way in which the function of the core pluripotency regulatory circuitry is integrated at a posttranslational level by controlling KLF4 protein stability. Furthermore, as we found that both SOX2 and NANOG regulated KLF4 only by posttranslational mechanisms, our findings contradict the accepted regulatory circuitry, which states that expression of these transcription factors is integrated by transcriptional mechanisms.

Nuclear localization and retention of KLF4 plays a key role in its regulation. KLF4 requires an intact NLS for nuclear import, as NLS mutant KLF4 protein is cytoplasmic (Dhaliwal et al. 2018). KLF4 nuclear localization increases protein stability, as the NLS mutant displayed greatly reduced stability compared with the WT protein. Mutations that disrupted KLF4 nuclear anchoring also disrupted KLF4 protein stability in LIF/2i conditions. Deletion of the KLF4 DNA-binding zinc finger domains or mutation of the K275 sumoylation site, involved in transactivation, caused a partial disruption to KLF4 nuclear anchoring. An important consequence of these mutations is that the stability of the protein was completely abolished, suggesting that even a small disruption to nuclear anchoring can greatly affect KLF4 protein stability. In contrast, in COS-1 cells, expression of a KLF4 K275 mutant did not affect protein stability, further indicating that regulation of KLF4 protein stability is context-dependent (Du et al. 2010). The transactivation function of KLF4 has been shown to be regulated in a SUMO1-dependent manner; mutation of the K275 sumoylation site in mouse KLF4 impairs the ability of KLF4 to transactivate target promoters by disrupting the SUMO-interacting motif in KLF4 (Du et al. 2010). We found that this mutation also reduced recruitment of KLF4 to RNAPII-rich nuclear compartments, which would explain reduced transactivation of the K275 mutant protein. Restoring nuclear anchoring in this transactivation mutant did not restore interaction with RNAPII, indicating that K275 is indeed required for interaction with RNAPII and KLF4 function.

ERK activation-induced KLF4 nuclear export is a critical first step in pluripotency exit, which relies on KLF4 phosphorylation at S132 and intact NES, allowing for interaction with XPO1 (Dhaliwal et al. 2018). We determined that monoubiquitination of KLF4 at K249 is also required for KLF4 nuclear export and pluripotency exit, although this event occurs later, after $24 \mathrm{~h}$ of differentiation. Three regions (S132, K249, and the KLF4 NES) are re- quired for the disruption to KLF4 stability that occurs as ES cells exit the pluripotent state, and preventing KLF4 phosphorylation ubiquitination, or interaction with XPO1 blocks pluripotency exit, which normally occurs after removal of $\mathrm{LIF} / 2 \mathrm{i}$. In contrast, even in the presence of LIF/2i, KLF4 stability can be disrupted by interfering with KLF4 function by deletion of the DNA-binding zinc finger domains or mutation of the K275 sumoylation site, which is involved in transactivation. The compound mutants (S132A $\triangle$ ZNF and S132AK275R), however, maintain KLF4 stability but not KLF4 regulatory activity or interaction with RNAPII and therefore do not block ES cell differentiation.

Naïve pluripotent ES cells cultured in $\mathrm{LIF} / 2 \mathrm{i}$ are thought to maintain a more balanced and stable state than ES cells maintained in LIF/serum through higher and more uniform expression of the major pluripotency transcription factors in the cell population (Tosolini and Jouneau 2016; Sim et al. 2017). Our results indicate that for KLF4 protein, this higher and more uniform expression in $L I F / 2 \mathrm{i}$ is achieved through posttranslational protein stabilization. This in turn could have knock-on effects leading to higher transcription of additional pluripotencyassociated transcription factors. Protein interactome studies determined that the core pluripotency transcription factors bind each other and form nuclear complexes in order to maintain the pluripotent state (Gao et al. 2012, 2013; Morey et al. 2015). KLF4 protein stability is maintained in ES cells through interaction of KLF4 with pSTAT3, NANOG, and SOX2 in RNAPII-rich nuclear complexes. Coimmunoprecipitation revealed that these interactions are maintained in the absence of both DNA and RNA, as these were removed by nuclease treatment during coimmunoprecipitation, suggesting that they are protein-protein interactions in nature. Even though KLF4-GFP coexpressed with SOX2, NANOG, and CASTAT3 in HEK293 cells displayed higher protein stability than KLF4-GFP expressed alone, the maximal HEK293 stability observed for KLF4-GFP $\left(t_{1 / 2} 3 \mathrm{~h}\right)$ remained lower than what was observed in undifferentiated ES cells $\left(t_{1 / 2}\right.$ $24 \mathrm{~h}$ ), suggesting that additional, unknown factors are involved in context-dependent stabilization of the KLF4 protein in pluripotent cells. KLF4 is exceptional in that the stability of other pluripotency factors or the other expressed Klf family members is not affected in the same way by these complexes, as their stability does not change during pluripotency exit. Although KLF4 is not required during mouse development (Segre et al. 1999), its downregulation through a disruption in protein stability is likely important during development, as we found that preventing protein destabilization blocks pluripotency exit and differentiation. Furthermore, the mutations to the KLF4 protein that increase KLF4 stability without affecting KLF4 function as a transcription factor could be deployed in a reprogramming context to improve the efficiency of reprogramming protocols by prolonging KLF4 protein function in reprogramming cells.

In addition to the role of KLF4 protein in pluripotency maintenance and reprogramming, KLF4 protein modulation is involved in both the oncogenic and tumor 
suppressor roles of KLF4 in adult tissues. Increased stability of the KLF4 protein, which is mediated by the ubiquitin-proteasomal pathway, is linked to an oncogenic role in breast cancer (Hu et al. 2012), and decreased stability is linked to its tumor suppressor role in colorectal cancer (Gamper et al. 2012). KLF4 stability modulation by interaction with the pluripotency transcription factors may also be important in tumorigenesis, as coexpression of these factors is associated with a variety of cancers such as breast cancer, squamous cell carcinoma, and gastrointestinal cancer progression ( $\mathrm{Hu}$ et al. 2012; Lu et al. 2014; Piva et al. 2014; Almozyan et al. 2017; Gwak et al. 2017; Soheili et al. 2017).

\section{Materials and methods}

ES cell culture

The mouse ES cell line E14TG2a (E14) was obtained from American Type Culture Collection (CRL-1821). F1 (M. musculus ${ }^{129} \times$ M. castaneus) ES cells were obtained from Barbara Panning (Mlynarczyk-Evans et al. 2006). All cells were maintained in feederfree conditions on $0.1 \%$ gelatin in DMEM supplemented with $15 \%(\mathrm{v} / \mathrm{v}$ ) fetal bovine serum (FBS), $0.1 \mathrm{mM}$ nonessential amino acids, $1 \mathrm{mM}$ sodium pyruvate, $2 \mathrm{mM}$ GlutaMAX, $0.1 \mathrm{mM}$ 2-mercaptoethanol, $1000 \mathrm{U} / \mathrm{mL}$ LIF, $3 \mu \mathrm{M}$ CHIR99021 /GSK3 $\beta$ inhibitor; Biovision), and $1 \mu \mathrm{M}$ PD0325901 (MEK inhibitor; Invitrogen), referred to as LIF/2i medium. Eighteen hours prior to differentiation, the cells were seeded at 10,000 cells per square centimeter. The differentiation medium contained the same components with the exception of LIF and the two inhibitors.

For protein half-life analysis, cells were treated with $10 \mu \mathrm{g} / \mathrm{mL}$ CHX (Sigma-Aldrich) for 2, 4 6, 8, and $12 \mathrm{~h}$; collected as cell pellets; and lysed in RIPA buffer containing protease inhibitor complete EDTA-free (Roche) and phosphatase inhibitor cocktail (Millipore) to generate cell lysates for further analysis by Western blotting. Treatment with $10 \mu \mathrm{M}$ MG132 proteasome inhibitor (Sigma-Aldrich) was used to indicate the role of proteasomal degradation in KLF4 function.

\section{Cellular fractionation, coimmunoprecipitation, and Western blotting}

Nuclear and cytoplasmic fractions were generated according to the protocol described in Dhaliwal and Mitchell (2016). Protein was extracted from cell fractions using RIPA buffer containing protease inhibitor complete EDTA-free (Roche) and phosphatase inhibitor cocktail (Millipore) and quantified using bicinchoninic acid (Thermo Fisher Scientific). Protein samples were analyzed by SDS-PAGE (Bis-Tris, 5\% stacking, 10\% resolving). Blots were probed with primary antibodies followed by horseradish peroxidase-conjugated secondary antibodies (Supplemental Table S1). Blots were quantified by relative intensity using background correction from adjacent regions. At least three biological replicates were analyzed for each experiment.

For coimmunoprecipitation of protein, fractions or total cell lysates in RIPA were treated with benzonase nuclease (Sigma-Aldrich) to remove RNA and DNA, incubated overnight with the appropriate antibody, and then incubated overnight with a 50:50 mixture of protein A and protein G Dynabeads (Thermo Fisher Scientific). Beads were washed three times with nondenaturing lysis buffer (20 mM Tris- $\mathrm{HCl}, 137 \mathrm{mM} \mathrm{NaCl}, 10 \%$ glycerol, 1\% NP-40, 2 mM EDTA, 1 mM PMSF, proteinase inhibitors) and twice with PBS, eluted in SDS-PAGE loading buffer, and analyzed by SDS-PAGE. The antibodies used are listed in Supplemental Table S1.
PLA

PLA was conducted using Duolink (Sigma-Aldrich) following the manufacturer's instructions. Images were collected using a Leica TCS SP8 and a $63 \times$ magnification objective lens. The number of PLA foci per nucleus was quantified using Imaris 7.1 by manual 3D masking of nuclei in ES cell colonies defined by the DAPI signal. All PLA experiments were carried out on at least three biological replicate samples.

\section{CRISPR/Cas9-mediated deletion}

Cas9-mediated deletions were carried out as described (Zhou et al. 2014; Moorthy and Mitchell 2016). Cas9 targeting guides flanking Klf4 enhancer regions were selected (Supplemental Table S2). Only gRNAs predicted to have no off-target binding in the F1 mouse genome were chosen. gRNA plasmids were assembled in the gRNA vector (Addgene, 41824) using the protocol described by Mali et al. (2013). The sequence of the resulting guide plasmid was confirmed by sequencing. F1 ES cells were transfected with $5 \mu \mathrm{g}$ each of $5^{\prime}$ gRNA, 3' gRNA, and pCas9_GFP (Addgene, 44719) (Ding et al. 2013) plasmids using the Neon transfection system (Life Technologies). Forty-eight hours after transfection, GFP-positive cells were collected and sorted on a BD FACSAria. GFP-positive cells $(10,000-20,000)$ were seeded on 10-cm gelatinized culture plates and grown for 5-6 d until large individual colonies formed. Colonies were picked and propagated for genotyping and gene expression analysis. All deletions were confirmed by sequence analysis using primers $5^{\prime}$ and $3^{\prime}$ from the gRNA target sites; SNPs within the amplified product confirmed the genotype of the deleted allele. Transcription factor binding at the Klf4 locus was determined by evaluating data obtained from CODEX (Sánchez-Castillo et al. 2015). ChIP-seq (chromatin immunoprecipitation [ChIP] coupled with highthroughput sequencing) data for KLF5 were obtained from Aksoy et al. (2014).

Real-time $q P C R$

Total RNA was purified as per the manufacturer's protocol using RNAeasy (Qiagen). Following a DNaseI (Turbo DNaseI from Invitrogen) digestion to remove DNA, total RNA was reversetranscribed with random primers using the high-capacity cDNA synthesis kit (Applied biosystems). Gene expression was monitored by qPCR using genomic DNA to generate standard curves. Gapdh expression was used to normalize expression values. At least three biological replicates were analyzed for each experiment. The primers used are listed in Supplemental Table S3. All samples were confirmed not to have DNA contamination by generating a reverse transcriptase-negative sample and monitoring Gapdh amplification. Allele-specific primers were determined to amplify only the specified allele by testing amplification on C57BL/6 (same genotype as 129 at the target sequence) and castaneus genomic DNA.

Expression of KLF4 mutants

A mouse KLF4-GFP vector (RG206691) obtained from Origene and a KLF4(S132A)-GFP mutant published in Dhaliwal et al. (2018) were subjected to site-directed mutagenesis (SDM; QuikChange Lightning, Agilent Technologies) to introduce additional mutations. Primers for SDM are indicated in Supplemental Table S4. A MluI/AleI restriction digestion deleted zinc fingers from the Klf4 sequence cloned in the pUC 19 vector. Klf4 in pUC19 without zinc fingers ( $3881 \mathrm{bp}$ ) was ligated by blunt end ligation. After sequence confirmation, the zinc finger-deleted Klf4 fragment was inserted into a Kpn1/Not1-digested KLF4-GFP vector. Sequence- 
confirmed plasmids were transfected by electroporation into E14 ES cells and selected with $400 \mu \mathrm{g} / \mathrm{mL}$ G418. The cells were sorted by fluorescence-activated cell sorting, and individual clones were selected and maintained in $50 \mu \mathrm{g} / \mathrm{mL}$ G418 to obtain KLF4-GFPpositive clones.

\section{Transient transfections}

Sox2-t2A-GFP was generated by amplifying the human SOX2 sequence from a donor construct (HsCD00079917, Harvard Institute of Proteomics) (Zuo et al. 2007) using NheI and XbaI overhang primers and Phusion high-fidelity polymerase (New England Biolabs). A t2a-GFP backbone was subcloned from pLV hU6-sgRNA hUbC-dCas9-KRAB-t2a-GFP (Addgene plasmid no. 71237, a gift from Charles Gersbach) (Thakore et al. 2015) and inserted into hCas9 (Addgene, plasmid no. 41815; a gift from George Church) (Mali et al. 2013) using AgeI/XbaI digestion and T4 DNA ligase (Thermo Fisher Scientific). Next, dCas9-KRAB was excised out using NheI/XbaI to create a lineralized CMVt2a-GFP construct. The SOX2 PCR product was purified and inserted into CMV-t2a-GFP using the In-Fusion HD cloning kit (Takara Bio USA) and transformed into Stellar-Competent cells (Takara Bio USA). To produce Sox2-t2A-mCherry, Sox2-t2AGFP was then cut with KpnI and AgeI to remove GFP and replace it with PCR-amplified mCherry from pAAVS1-NDi-CRISPRi (Addgene, plasmid no. 73497; a gift from Bruce Conklin) (Mandegar et al. 2016) with KpnI and AgeI overhang primers. Sox2-t2AmCherry was digested with XbaI and NheI to remove Sox2 and replace it with PCR products from Nanog (Addgene, plasmid no. 59994; a gift from Rudolf Jaenisch) (Faddah et al. 2013), Klf2 (Addgene, plasmid no. 66655; a gift from Barak Cohen), and CAStat3 (Addgene, plasmid no. 8722; a gift from Jim Darnell) (Bromberg et al. 1999). Bacterial colonies were PCR-screened, and positive inserts were sequence-confirmed. All plasmids were purified with an endotoxin-free plasmid Midiprep kit (Geneaid Midi plasmid kit endotoxin-free).

The SOX2-compromised SCR ${ }^{129 / \text { Cast }}$-deleted cells were transfected with Sox2-t2A-GFP, and 24-h differentiated ES cells were transfected with Nanog-t2A-GFP using neon electroporation transfection system as per the manufacturer's instructions (Thermo Fisher Scientific). Nanog-2A-GFP was a gift from Rudolf Jaenisch (Addgene, plasmid no. 59994) (Faddah et al. 2013).

HEK293 (Flp-In-293 cell line, Thermo Fisher Scientific) cells were grown in DMEM supplemented with $10 \%$ FBS, $1 \times$ pennicillin-streptomycin, $1 \times$ glutamax, $1 \times$ nonessential amino acids, and $1 \times$ sodium pyruvate. Cells were split into six-well plates and seeded at 30,000 cells per square centimeter $18 \mathrm{~h}$ before transfection. KLF4-GFP was transfected alone or with Klf2-t2A-mCherry, Sox2-t2A-mCherry, CA-Stat3-t2A-mCherry, or Nanog-t2AmCherry in different combinations at $2 \mu \mathrm{g}$ per well. Lipofectamine 3000 at $0.3 \mu \mathrm{L}$ per microliter of Opti-MEM (Thermo Fisher Scientific) was mixed with plasmids at $1.5 \mu \mathrm{L}$ per microgram of plasmid. Transfected cells were treated with $10 \mu \mathrm{g} / \mathrm{mL}$ CHX (Sigma-Aldrich) and sampled after 0, 2, 4, 6, 8, and $12 \mathrm{~h}$.

Vectors constructed for transient transfections are available through Addgene.

\section{Acknowledgments}

This work was supported by the Canadian Institutes of Health Research (FRN 153186), the Canada Foundation for Innovation, and the Ontario Ministry of Research and Innovation (operating and infrastructure grants held by J.A.M.). Studentship funding was provided by Ontario Graduate Scholarships held by N.K.D.
Author contributions: J.A.M. and N.K.D. conceived and designed the experiments. N.K.D. performed all experiments with the following assistance: L.E.A. constructed several of the vectors used in this study and analyzed KLF5 ChIP-seq data. N.K.D. and J.A.M. analyzed the data and wrote the manuscript, which was approved by all coauthors.

\section{References}

Aksoy I, Giudice V, Delahaye E, Wianny F, Aubry M, Mure M, Chen J, Jauch R, Bogu GK, Nolden T, et al. 2014. Klf4 and Klf5 differentially inhibit mesoderm and endoderm differentiation in embryonic stem cells. Nat Commun 5: 3719. doi:10 $.1038 /$ ncomms4719

Almozyan S, Colak D, Mansour F, Alaiya A, Al-Harazi O, Qattan A, Al-Mohanna F, Al-Alwan M, Ghebeh H. 2017. PD-L1 promotes OCT4 and Nanog expression in breast cancer stem cells by sustaining PI3K/AKT pathway activation. Int J Cancer 141: 1402-1412. doi:10.1002/ijc.30834

Blinka S, Reimer MH Jr, Pulakanti K, Rao S. 2016. Super-enhancers at the Nanog locus differentially regulate neighboring pluripotency-associated genes. Cell Rep 17: 19-28. doi:10.1016/j .celrep.2016.09.002

Bromberg JF, Wrzeszczynska MH, Devgan G, Zhao Y, Pestell RG, Albanese C, Darnell JE Jr. 1999. Stat3 as an oncogene. Cell 98: 295-303. doi:10.1016/S0092-8674(00)81959-5

Carter D, Chakalova L, Osborne CS, Dai YF, Fraser P. 2002. Longrange chromatin regulatory interactions in vivo. Nat Genet 32: 623-626. doi:10.1038/ng1051

Chen X, Xu H, Yuan P, Fang F, Huss M, Vega VB, Wong E, Orlov YL, Zhang W, Jiang J, et al. 2008. Integration of external signaling pathways with the core transcriptional network in embryonic stem cells. Cell 133: 1106-1117. doi:10.1016/j.cell.2008 .04 .043

Chen CY, Morris Q, Mitchell JA. 2012. Enhancer identification in mouse embryonic stem cells using integrative modeling of chromatin and genomic features. BMC Genomics 13: 152. doi:10.1186/1471-2164-13-152

Dhaliwal NK, Mitchell JA. 2016. Nuclear RNA isolation and sequencing. Methods Mol Biol 1402: 63-71. doi:10.1007/978-14939-3378-5_7

Dhaliwal NK, Miri K, Davidson S, Tamim El Jarkass H, and Mitchell JA. 2018. KLF4 nuclear export requires ERK activation and initiates exit from naive pluripotency. Stem Cell Reports 10: 1308-1323. doi:10.1016/j.stemcr.2018.02.007

Ding Q, Regan SN, Xia Y, Oostrom LA, Cowan CA, Musunuru K. 2013. Enhanced efficiency of human pluripotent stem cell genome editing through replacing TALENs with CRISPRs. Cell Stem Cell 12: 393-394. doi:10.1016/j.stem.2013.03.006

Du JX, McConnell BB, Yang VW. 2010. A small ubiquitin-related modifier-interacting motif functions as the transcriptional activation domain of Krüppel-like factor 4. J Biol Chem 285: 28298-28308. doi:10.1074/jbc.M110.101717

Faddah DA, Wang H, Cheng AW, Katz Y, Buganim Y, Jaenisch R. 2013. Single-cell analysis reveals that expression of nanog is biallelic and equally variable as that of other pluripotency factors in mouse ESCs. Cell Stem Cell 13: 23-29. doi:10.1016/j .stem.2013.04.019

Gamper AM, Qiao X, Kim J, Zhang L, DeSimone MC, Rathmell WK, Wan Y. 2012. Regulation of KLF4 turnover reveals an unexpected tissue-specific role of pVHL in tumorigenesis. Mol Cell 45: 233-243. doi:10.1016/j.molcel.2011.11.031

Gao Z, Cox JL, Gilmore JM, Ormsbee BD, Mallanna SK, Washburn MP, Rizzino A. 2012. Determination of protein 
interactome of transcription factor Sox 2 in embryonic stem cells engineered for inducible expression of four reprogramming factors. I Biol Chem 287: 11384-11397. doi:10.1074/ jbc.M111.320143

Gao F, Wei Z, An W, Wang K, Lu W. 2013. The interactomes of POU5F1 and SOX2 enhancers in human embryonic stem cells. Sci Rep 3: 1588. doi:10.1038/srep01588

Gwak JM, Kim M, Kim HI, Jang MH, Park SY. 2017. Expression of embryonal stem cell transcription factors in breast cancer: Oct 4 as an indicator for poor clinical outcome and tamoxifen resistance. Oncotarget 8: 36305-36318.

Hall J, Guo G, Wray J, Eyres I, Nichols J, Grotewold L, Morfopoulou S, Humphreys P, Mansfield W, Walker R, et al. 2009. Oct4 and LIF/Stat3 additively induce Krüppel factors to sustain embryonic stem cell self-renewal. Cell Stem Cell 5: 597-609. doi:10.1016/j.stem.2009.11.003

Hirai H, Karian P, Kikyo N. 2011. Regulation of embryonic stem cell self-renewal and pluripotency by leukaemia inhibitory factor. Biochem J 438: 11-23. doi:10.1042/BJ20102152

Hochstrasser M, Varshavsky A. 1990. In vivo degradation of a transcriptional regulator: the yeast a 2 repressor. Cell 61: 697-708. doi:10.1016/0092-8674(90)90481-S

Hu D, Zhou Z, Davidson NE, Huang Y, Wan Y. 2012. Novel insight into KLF4 proteolytic regulation in estrogen receptor signaling and breast carcinogenesis. I Biol Chem 287: 1358413597. doi:10.1074/jbc.M112.343566

Jovanovic M, Rooney MS, Mertins P, Przybylski D, Chevrier N, Satija R, Rodriguez EH, Fields AP, Schwartz S, Raychowdhury $\mathrm{R}$, et al. 2015. Immunogenetics. Dynamic profiling of the protein life cycle in response to pathogens. Science 347: 1259038. doi:10.1126/science.1259038

Keşmir C, Nussbaum AK, Schild H, Detours V, Brunak S. 2002. Prediction of proteasome cleavage motifs by neural networks. Protein Eng 15: 287-296. doi:10.1093/protein/15.4.287

Kim MO, Kim SH, Cho YY, Nadas J, Jeong CH, Yao K, Kim DJ, Yu DH, Keum YS, Lee KY, et al. 2012. ERK1 and ERK2 regulate embryonic stem cell self-renewal through phosphorylation of Klf4. Nat Struct Mol Biol 19: 283-290. doi:10.1038/nsmb .2217

Kim SH, Kim MO, Cho YY, Yao K, Kim DJ, Jeong CH, Yu DH, Bae KB, Cho EJ, Jung SK, et al. 2014. ERK1 phosphorylates Nanog to regulate protein stability and stem cell self-renewal. Stem Cell Res 13: 1-11. doi:10.1016/j.scr.2014.04.001

Kristensen AR, Gsponer J, Foster LJ. 2013. Protein synthesis rate is the predominant regulator of protein expression during differentiation. Mol Syst Biol 9: 689. doi:10.1038/msb.2013.47

Li Y, Rivera CM, Ishii H, Jin F, Selvaraj S, Lee AY, Dixon JR, Ren B. 2014. CRISPR reveals a distal super-enhancer required for Sox2 expression in mouse embryonic stem cells. PLoS One 9: e114485. doi:10.1371/journal.pone.0114485

Lim KH, Kim SR, Ramakrishna S, Baek KH. 2014. Critical lysine residues of Klf4 required for protein stabilization and degradation. Biochem Biophys Res Commun 443: 1206-1210. doi:10 .1016/j.bbrc.2013.12.121

Liu Y, Beyer A, Aebersold R. 2016. On the dependency of cellular protein levels on mRNA abundance. Cell 165: 535-550. doi:10 .1016/j.cell.2016.03.014

Lu X, Mazur SJ, Lin T, Appella E, Xu Y. 2014. The pluripotency factor nanog promotes breast cancer tumorigenesis and metastasis. Oncogene 33: 2655-2664. doi:10.1038/onc.2013.209

Mali P, Yang L, Esvelt KM, Aach J, Guell M, DiCarlo JE, Norville JE, Church GM. 2013. RNA-guided human genome engineering via Cas9. Science 339: 823-826. doi:10.1126/science .1232033
Mandegar MA, Huebsch N, Frolov EB, Shin E, Truong A, Olvera MP, Chan AH, Miyaoka Y, Holmes K, Spencer CI, et al. 2016. CRISPR interference efficiently induces specific and reversible gene silencing in human iPSCs. Cell Stem Cell 18: 541553. doi:10.1016/j.stem.2016.01.022

Matsuda T, Nakamura T, Nakao K, Arai T, Katsuki M, Heike T, Yokota T. 1999. STAT3 activation is sufficient to maintain an undifferentiated state of mouse embryonic stem cells. EMBO I 18: 4261-4269. doi:10.1093/emboj/18.15.4261

Mlynarczyk-Evans S, Royce-Tolland M, Alexander MK, Andersen AA, Kalantry S, Gribnau J, Panning B. 2006. X chromosomes alternate between two states prior to random Xinactivation. PLOS Biol 4: e159. doi:10.1371/journal.pbio .0040159

Moorthy SD, Mitchell JA. 2016. Generating CRISPR/Cas9 mediated monoallelic deletions to study enhancer function in mouse embryonic stem cells. J Vis Exp doi:10.3791/53552.

Moorthy SD, Davidson S, Shchuka VM, Singh G, Malek-Gilani N, Langroudi L, Martchenko A, So V, Macpherson NN, Mitchell JA. 2017. Enhancers and super-enhancers have an equivalent regulatory role in embryonic stem cells through regulation of single or multiple genes. Genome Res 27: 246258. doi:10.1101/gr.210930.116

Morey L, Santanach A, Di Croce L. 2015. Pluripotency and epigenetic factors in mouse embryonic stem cell fate regulation. Mol Cell Biol 35: 2716-2728. doi:10.1128/MCB.00266-15

Nichols J, Smith A. 2009. Naive and primed pluripotent states. Cell Stem Cell 4: 487-492. doi:10.1016/j.stem.2009.05.015

Niwa H, Burdon T, Chambers I, Smith A. 1998. Self-renewal of pluripotent embryonic stem cells is mediated via activation of STAT3. Genes Dev 12: 2048-2060. doi:10.1101/gad.12.13 .2048

Ohtsuka S, Nakai-Futatsugi Y, Niwa H. 2015. LIF signal in mouse embryonic stem cells. JAKSTAT 4: e1086520.

Piva M, Domenici G, Iriondo O, Rabano M, Simoes BM, Comaills V, Barredo I, Lopez-Ruiz JA, Zabalza I, Kypta R, et al. 2014. Sox 2 promotes tamoxifen resistance in breast cancer cells. EMBO Mol Med 6: 66-79. doi:10.1002/emmm.201303411

Radivojac P, Vacic V, Haynes C, Cocklin RR, Mohan A, Heyen JW, Goebl MG, Iakoucheva LM. 2010. Identification, analysis, and prediction of protein ubiquitination sites. Proteins 78: 365-380. doi:10.1002/prot.22555

Raz R, Lee CK, Cannizzaro LA, d'Eustachio P, Levy DE. 1999. Essential role of STAT3 for embryonic stem cell pluripotency. Proc Natl Acad Sci 96: 2846-2851. doi:10.1073/pnas.96.6 .2846

Sánchez-Castillo M, Ruau D, Wilkinson AC, Ng FS, Hannah R, Diamanti E, Lombard P, Wilson NK, Gottgens B. 2015. CODEX: a next-generation sequencing experiment database for the haematopoietic and embryonic stem cell communities. Nucleic Acids Res 43: D1117-D1123. doi:10.1093/nar/ gku895

Schoenfelder S, Furlan-Magaril M, Mifsud B, Tavares-Cadete F, Sugar R, Javierre BM, Nagano T, Katsman Y, Sakthidevi M, Wingett SW, et al. 2015. The pluripotent regulatory circuitry connecting promoters to their long-range interacting elements. Genome Res 25: 582-597. doi:10.1101/gr.185272.114

Schuetz A, Nana D, Rose C, Zocher G, Milanovic M, Koenigsmann J, Blasig R, Heinemann U, Carstanjen D. 2011. The structure of the Klf4 DNA-binding domain links to self-renewal and macrophage differentiation. Cell Mol Life Sci 68: 3121-3131. doi:10.1007/s00018-010-0618-x

Segre JA, Bauer C, Fuchs E. 1999. Klf4 is a transcription factor required for establishing the barrier function of the skin. Nat Genet 22: 356-360. doi:10.1038/11926 
Sim YJ, Kim MS, Nayfeh A, Yun YJ, Kim SJ, Park KT, Kim CH, Kim KS. 2017. 2i maintains a naive ground state in ESCs through two distinct epigenetic mechanisms. Stem Cell Reports 8: 1312-1328. doi:10.1016/j.stemcr.2017.04.001

Soheili S, Asadi MH, Farsinejad A. 2017. Distinctive expression pattern of OCT4 variants in different types of breast cancer. Cancer Biomark 18: 69-76. doi:10.3233/CBM-160675

Thakore PI, D'Ippolito AM, Song L, Safi A, Shivakumar NK, Kabadi AM, Reddy TE, Crawford GE, Gersbach CA. 2015. Highly specific epigenome editing by CRISPR-Cas9 repressors for silencing of distal regulatory elements. Nat Methods 12: 1143-1149. doi:10.1038/nmeth.3630

Theunissen TW, van Oosten AL, Castelo-Branco G, Hall J, Smith A, Silva JC. 2011. Nanog overcomes reprogramming barriers and induces pluripotency in minimal conditions. Curr Biol 21: 65-71. doi:10.1016/j.cub.2010.11.074

Tolhuis B, Palstra RJ, Splinter E, Grosveld F, de Laat W. 2002. Looping and interaction between hypersensitive sites in the active $\beta$-globin locus. Mol Cell 10: 1453-1465. doi:10.1016/ S1097-2765(02)00781-5

Tosolini M, Jouneau A. 2016. Acquiring ground state pluripotency: switching mouse embryonic stem cells from serum/ LIF medium to 2i/LIF medium. Methods Mol Biol 1341: 4148. doi:10.1007/7651_2015_207

Visel A, Blow MJ, Li Z, Zhang T, Akiyama JA, Holt A, PlajzerFrick I, Shoukry M, Wright C, Chen F, et al. 2009. ChIP-seq accurately predicts tissue-specific activity of enhancers. Nature 457: 854-858. doi:10.1038/nature07730

Wei Z, Yang Y, Zhang P, Andrianakos R, Hasegawa K, Lyu J, Chen X, Bai G, Liu C, Pera M, et al. 2009. Klf4 interacts directly with Oct4 and Sox 2 to promote reprogramming. Stem Cells 27: 2969-2978.
Wei Z, Gao F, Kim S, Yang H, Lyu J, An W, Wang K, Lu W. 2013. Klf4 organizes long-range chromosomal interactions with the Oct4 locus in reprogramming and pluripotency. Cell Stem Cell 13: 36-47. doi:10.1016/j.stem.2013.05.010

Wray J, Kalkan T, Smith AG. 2010. The ground state of pluripotency. Biochem Soc Trans 38: 1027-1032. doi:10.1042/ BST0381027

Xie L, Torigoe SE, Xiao J, Mai DH, Li L, Davis FP, Dong P, MarieNelly H, Grimm J, Lavis L, et al. 2017. A dynamic interplay of enhancer elements regulates Klf4 expression in naïve pluripotency. Genes Dev 31: 1795-1808. doi:10.1101/gad.303321.117

Yeom YI, Fuhrmann G, Ovitt CE, Brehm A, Ohbo K, Gross M, Hubner K, Scholer HR. 1996. Germline regulatory element of Oct-4 specific for the totipotent cycle of embryonal cells. Development 122: 881-894.

Zhang P, Andrianakos R, Yang Y, Liu C, Lu W. 2010. Kruppel-like factor 4 (Klf4) prevents embryonic stem (ES) cell differentiation by regulating Nanog gene expression. J Biol Chem 285: 9180-9189. doi:10.1074/jbc.M109.077958

Zhou BP, Deng J, Xia W, Xu J, Li YM, Gunduz M, Hung MC. 2004. Dual regulation of Snail by GSK-3 $\beta$-mediated phosphorylation in control of epithelial-mesenchymal transition. Nat Cell Biol 6: 931-940. doi:10.1038/ncb1173

Zhou HY, Katsman Y, Dhaliwal NK, Davidson S, Macpherson NN, Sakthidevi M, Collura F, Mitchell JA. 2014. A Sox2 distal enhancer cluster regulates embryonic stem cell differentiation potential. Genes Dev 28: 2699-2711. doi:10.1101/gad .248526 .114

Zuo D, Mohr SE, Hu Y, Taycher E, Rolfs A, Kramer J, Williamson J, LaBaer J. 2007. PlasmID: a centralized repository for plasmid clone information and distribution. Nucleic Acids Res 35: D680-D684. doi:10.1093/nar/gk1898 


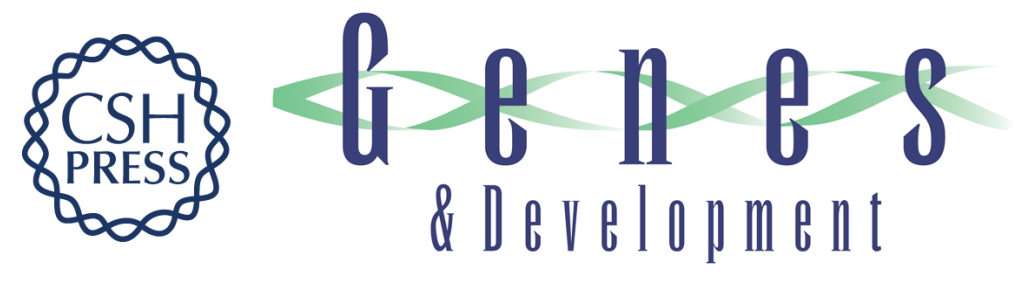

\title{
KLF4 protein stability regulated by interaction with pluripotency transcription factors overrides transcriptional control
}

\author{
Navroop K. Dhaliwal, Luis E. Abatti and Jennifer A. Mitchell
}

Genes Dev. 2019, 33: originally published online June 20, 2019

Access the most recent version at doi:10.1101/gad.324319.119

\section{Supplemental http://genesdev.cshlp.org/content/suppl/2019/06/14/gad.324319.119.DC1 Material}

References This article cites 62 articles, 19 of which can be accessed free at: http://genesdev.cshlp.org/content/33/15-16/1069.full.html\#ref-list-1

Creative This article is distributed exclusively by Cold Spring Harbor Laboratory Press for the first Commons six months after the full-issue publication date (see

License http://genesdev.cshlp.org/site/misc/terms.xhtml). After six months, it is available under a Creative Commons License (Attribution-NonCommercial 4.0 International), as described at http://creativecommons.org/licenses/by-nc/4.0/.

Email Alerting Receive free email alerts when new articles cite this article - sign up in the box at the top Service right corner of the article or click here.

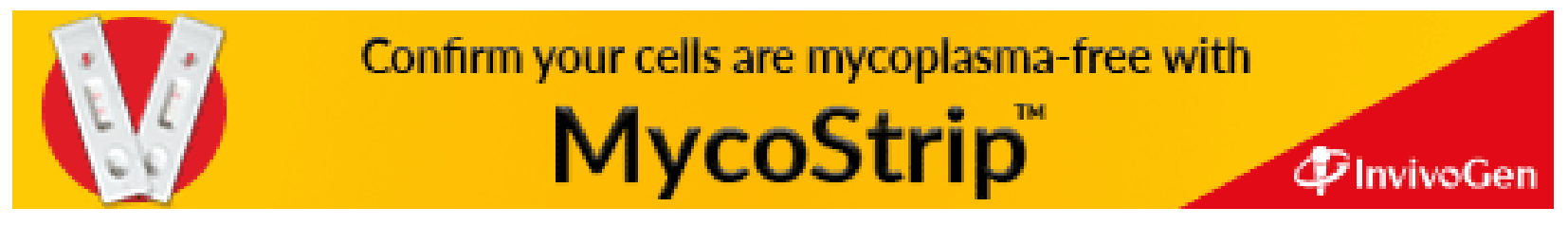

